ARTICLE

\title{
Epistasis shapes the fitness landscape of an allosteric specificity switch
}

Kyle K. Nishikawa', Nicholas Hoppe (1) ${ }^{1}$, Robert Smith¹, Craig Bingman (1) ${ }^{1}$ \& Srivatsan Raman (D) 1,2,3凶

Epistasis is a major determinant in the emergence of novel protein function. In allosteric proteins, direct interactions between inducer-binding mutations propagate through the allosteric network, manifesting as epistasis at the level of biological function. Elucidating this relationship between local interactions and their global effects is essential to understanding evolution of allosteric proteins. We integrate computational design, structural and biophysical analysis to characterize the emergence of novel inducer specificity in an allosteric transcription factor. Adaptive landscapes of different inducers of the designed mutant show that a few strong epistatic interactions constrain the number of viable sequence pathways, revealing ridges in the fitness landscape leading to new specificity. The structure of the designed mutant shows that a striking change in inducer orientation still retains allosteric function. Comparing biophysical and functional properties suggests a nonlinear relationship between inducer binding affinity and allostery. Our results highlight the functional and evolutionary complexity of allosteric proteins. 
nteractions between mutations direct the evolution of protein function ${ }^{1}$. As proteins evolve, they follow paths through the fitness landscape to reach a fitness peak that represents a novel function ${ }^{2}$. For $\mathrm{N}$ mutations required to confer novel function, there are $\mathrm{N}$ ! possible pathways connecting the start and end states. However, some pathways may not be evolutionarily favorable due to epistasis-a phenomenon that occurs when the sequence background into which a mutation is introduced changes the functional effect of that mutation. The non-additivity due to epistasis strongly influences the sequence trajectory a protein takes to gain new function ${ }^{1,3-5}$. Therefore, understanding the nature of epistatic interactions is the foundation for investigating the mechanisms leading to novel protein function ${ }^{6}$.

Epistasis is generally categorized as specific or nonspecific based on cause-effect relationships between the interactions of mutations and their outcome. Specific epistasis occurs between a limited number of residues that typically physically interact, leading to nonadditive changes in thermodynamically driven biophysical properties such as protein stability or affinity ${ }^{7}$. Specific epistasis has been extensively investigated in protein-protein, protein-ligand, protein-DNA interactions ${ }^{5,8-16}$. Nonspecific epistasis occurs when mutations are nonadditive with respect to protein traits when combined ${ }^{17-20}$. Such mutations can be spatially distant such as a global suppressor that can interact with many destabilizing mutations with low pairing specificity ${ }^{4,21,22}$.

In this study, we examine the role of epistasis in the evolution of ligand specificity in an allosteric transcription factor. Allostery is a fundamental mechanism by which proteins recognize environmental cues (such as binding of an inducer or effector) within a localized region resulting in modulation of function at a distal site 23,24 . Mutations in the binding pocket that trigger the allosteric network have the potential to create new nonspecific epistatic interactions at the level of protein function, beyond the physical interactions commonly seen in specific epistasis. As allosteric proteins evolve toward new function, such as orthologs in different organisms, their inducer specificity changes to adapt to the new environment ${ }^{25}$. Allosteric proteins may accrue mutations during evolution that would simultaneously affect specificities for old and new inducers. Further, these mutations may also impact function by affecting the capability of the protein to produce an allosteric change in response to an inducer ${ }^{26,27}$. For an allosteric transcription factor (aTF), a function is the outcome of affinity for the inducer ligand, affinity for DNA, and allosteric changes that accompany binding to the ligand. Each of these parameters will have its own fitness function mapped over the same sequence space, creating unique fitness landscapes. An aTF simultaneously traverses these multiple fitness landscapes, which collectively govern the evolutionary trajectory of the aTF under selective pressure. Thus, any one fitness landscape is not adequate as a global measure of transcription factor function. We need to examine multiple fitness landscapes and characterize epistasis in each to understand the evolutionary trajectory of an aTF.

Here, we integrate functional, structural, and biophysical analysis to characterize epistasis in the functional parameters of an allosteric transcription factor. Using computation-guided design, we changed the ligand specificity of TtgR, a microbial aTF, to respond better to one of its native ligands (resveratrol), but not to another (naringenin) by targeting mutations to positions that directly interact with the ligand to create a resveratrol-specific TtgR variant ${ }^{28,29}$. By reconstructing all sequence pathways connecting the two states, we found that nonspecific epistatic interactions of two distinct sets of amino acids separately drive loss of naringenin response while increasing resveratrol response (reporter expression when induced by a ligand). We characterized the fitness landscapes of TtgR in terms of four functional parameters: fold change in gene expression, basal gene expression, maximum gene expression, and sensitivity to the ligand $\left(\mathrm{EC}_{50}\right)$ and showed that although ligandinduced allostery is a composite effect of all four parameters, each parameter shows unique patterns of epistasis, but also notable similarities. The crystal structure of the computationally designed mutant shows that one of the mutations reshapes the binding pocket to favor resveratrol over naringenin through a striking change in its binding orientation while maintaining allostery. We found that epistasis creates distinct biophysical and biological functional landscapes. Our results highlight the functional and evolutionary complexity of allosteric proteins because pathways can traverse through multiple adaptive landscapes under evolutionary pressure 29 . Our approach also provides a general conceptual and methodological framework to investigate epistasis in transcription factors.

\section{Results}

Computational design of ligand specificity switch. We chose TtgR, a ligand-inducible aTF belonging to the diverse TetR-like protein family, as a target for computational engineering of ligand specificity ${ }^{29}$. $\operatorname{TtgR}$ is a 1 -component transcriptional system and represents the simplest molecular mechanism for converting biophysical interaction between inducer and protein into a complex biological response like transcription ${ }^{29}$. In the uninduced state, TtgR physically obstructs the RNA polymerase by binding to $\mathrm{DNA}^{29}$. When induced, ligand-binding allosterically lowers affinity for DNA, thereby allowing transcription ${ }^{29,30}$. Since TtgR is found in a plant-associated microbe (Pseudomonas putida), it is induced by multiple plant molecules including resveratrol and naringenin ${ }^{28}$. Thus, $\mathrm{TtgR}$ provides a suitable functional backdrop to investigate the role of epistasis in the emergence of novel function (ligand specificity) in an allosteric protein $^{28,31}$. To emulate the emergence of novel function, we engineered $\operatorname{TtgR}$ to respond better to resveratrol and not to naringenin.

We used computational design (Rosetta software suite) to engineer TtgR specificity by generating function-switching mutations that directly interact with the ligand ${ }^{32}$. Less directed approaches may yield a specificity switch, but these can also include distal mutations whose effects on ligand affinity will confound our examination of epistasis ${ }^{10,33}$. Since our goal was to study how local interactions shape global function, computational design was the appropriate tool as in silico mutations are chosen based on interaction energies between protein and ligand ${ }^{34,35}$.

To increase resveratrol specificity, we redesigned the ligandcontacting residues for greater affinity for resveratrol, assuming greater affinity may result in greater specificity. Since Rosetta is a structure-based design tool, the absence of a resveratrol-bound TtgR crystal structure made the design task challenging because the correct position of the ligand in the binding pocket was not known a priori. Therefore, we generated a set of diverse starting poses (16) by docking resveratrol conformers in different orientations within the binding pocket (Fig. 1). For each starting pose, we redesigned ligand-contacting residues while permitting constrained rigid-body flexibility of the ligand and torsional flexibility of the protein backbone. We computationally generated approximately 19,000 unique TtgR design variants. After design, each output variant comes with a set of Rosetta-calculated scores that reflect physical properties such as stability, repulsion, hydrogen bonds, and protein-ligand affinity. The best variants for library construction can be selected from the distribution of all scores of output designs based on user-defined preferences. The variants were curated using parameter-specific median absolute deviation cutoffs on a select set of Rosetta scoring metrics to yield a final list of approximately 3500 unique sequences with an average of five mutations per variant for experimental testing 

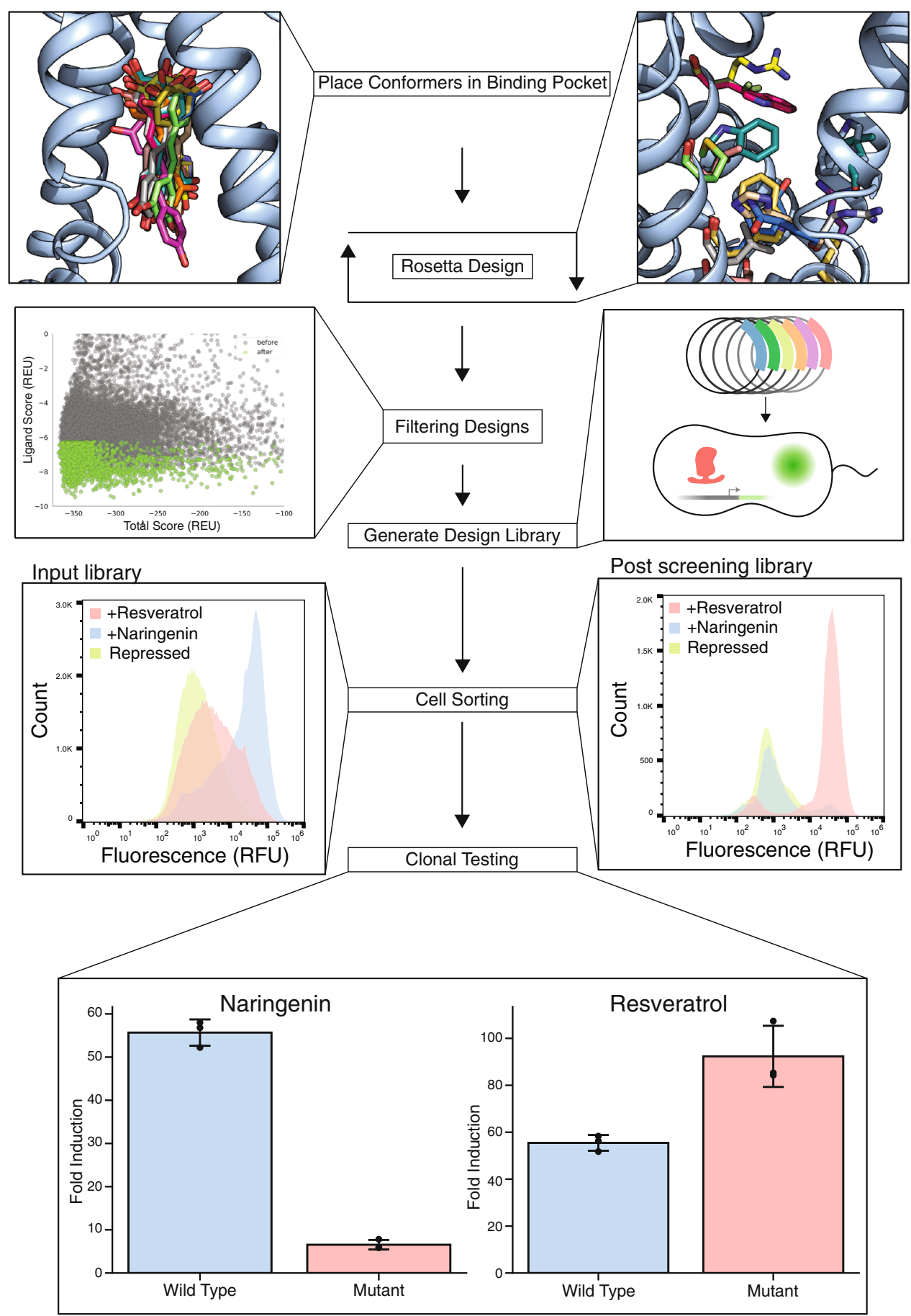

Fig. 1 Design of resveratrol-specific TtgR variant. Resveratrol conformers are docked into TtgR followed by Rosetta-based computational design of the binding pocket. Candidates with favorable Rosetta score metrics (green points) are synthesized and cloned into an expression vector. Distribution of fluorescence in cells containing uninduced TtgR variant library (light green), induced with naringenin (light blue) and resveratrol (red) before sorting (PreSort) and after three rounds of sorting (Post-Sort) are shown. Colony screening identified a quadruple mutant showing resveratrol specificity: C137l/ 1141W/M167L/F168Y. The quadruple mutant phenotype was compared to wild-type in biological triplicate $(n=3)$ by inducing each with either $1000 \mu \mathrm{M}$ naringenin or $100 \mu \mathrm{M}$ resveratrol. The error bars denote the standard deviation of the fold induction for the triplicate measurements (see "methods").

(Supplementary Figs. 1, 2). The mutations generated in the 3500 sequences are diverse, but designed sequences generally favor the wild-type amino acid at each mutable position (Supplementary Fig. 2). A few positions such as 96, 137, 168, and 175 have mutations that are more abundant than the wildtype amino acid. We synthesized oligonucleotides encoding approximately 3500 designed variants as a pool of exact chipDNA sequences (Twist Bioscience Inc).
To determine the activity of TtgR variants, we designed a pooled screen by sorting E. coli cells containing a GFP reporter system regulated by a TtgR operator adapted for E. coli. We quantified the activity of variants based on fold induction: the ratio of GFP expression with and without inducer. Fold induction is a simple measure of the transcriptional activity of an aTF that accounts for factors affected by epistasis including DNA affinity, ligand affinity and allostery ${ }^{5,9}$. The activity of the initial library 
was greater toward naringenin than resveratrol with a median fold induction of 21-fold and 2.4-fold with naringenin and resveratrol, respectively (Fig. 1). To enrich resveratrol-specific variants in the library, we devised a toggled screening scheme where we first sorted variants competent for binding to DNA (low GFP with no resveratrol) followed by sorting variants that can activate expression of the reporter (high GFP with resveratrol) (Supplementary Fig. 3). After three rounds of toggled screening, we observed a much greater response to resveratrol than naringenin in the enriched population compared to the input population (Fig.1). From the enriched population, we isolated a resveratrol-specific TtgR variant with four mutations: C137I, I141W, M167L, and F168Y which we will henceforth refer to as the 'quadruple mutant'. All four mutations were in close proximity to the ligand and no mutations were found elsewhere on TtgR. The quadruple mutant gave 92- and 6.5-fold induction with $100 \mu \mathrm{M}$ resveratrol and $1 \mathrm{mM}$ naringenin, respectively, compared to 55- and 55-fold of wild-type TtgR (Fig. 1, Supplementary Fig. 4). These concentrations were selected based on the differences in maximum solubility in aqueous solution.

The goal of Rosetta design was to narrow the potential designable sequence space to a subspace of sequences most likely to offer high resveratrol function. It is possible that other Rosetta designs were successful in generating ligand specificity but were lost in the screening process that was engineered to identify only the most successful variants. We found that while the quadruple mutant fell within the cutoffs imposed during the curation process, it was not the best in any scoring parameter. We chose the quadruple mutant as the functional endpoint for characterizing epistasis.

Epistasis shapes the fitness landscape of resveratrol response. Experimental fitness landscapes are a useful framework for characterizing epistasis by revealing fitness pathways through mutational intermediates that connect two functional states. We constructed multiple fitness landscapes derived from dose-response curves to examine epistatic constraints in the transition from wildtype TtgR to the resveratrol-specific quadruple mutant. We made all single, double, and triple mutation combinations of the four mutations that provide resveratrol specificity as individual clones, resulting in a total of 16 variants (including endpoints). Fitness landscapes are commonly illustrated as a series of nodes and edges. Each node is designated by a binary string in which each number corresponds to a mutable position. A zero indicates the wild-type amino acid identity and a one indicates the substituted amino acid. The positions in order from left to right are 137, 141, 167, and 168 (0000 is wild-type TtgR, 1111 is quadruple mutant, and 0100 represents the I141W mutant).

The ability of a transcription factor to control gene expression in response to a small molecule is broadly described by four parameters-(1) fold change in gene expression upon induction (fold induction), (2) basal gene expression without the inducer, (3) maximum gene expression upon induction, and (4) sensitivity to ligand concentration (EC). These parameters capture the mechanistic properties of binding to inducer, binding to DNA, and allosteric communication of ligand binding. To investigate how the same set of binding pocket mutations might uniquely affect each parameter, we constructed the fitness landscape of each parameter individually. We quantified the number of viable pathways in the resveratrol landscape by requiring that each additional mutation must increase parameter fitness if the quadruple mutant performs better than wild type or decrease parameter fitness if the quadruple mutant performs worse than wild type. There are 24 possible pathways from wild type to quadruple mutant (Fig. 2a). Each functional parameter shows distinctive patterns of epistasis, although some are closely related.

In the fold induction landscape, viable pathways must go through 0010 as all other single mutants have lower resveratrol response relative to wild-type TtgR (Fig. 2a). This restricts the number of available pathways from 24 to a maximum of 6 . From 0010, there are three possible double mutants: 0011,0110, and 1010. Both 0110 and 0011 are not viable as their activity substantially decreases compared to 0010 (Fig. 2a). However, 1010 is viable as it gives modestly higher resveratrol response (Fig. 2a). Both C137I and M167L manifest as key permissive intermediates in the fitness landscape that allows I141W (1110) or F168Y (1011) to be added. Since 1010 is the only viable double mutant, the number of available pathways reduces to two (Fig. 2a, bold red lines). Both triple mutants (1011 and 1110) have higher resveratrol response than 1010 which allows two viable pathways to reach the quadruple mutant, which is the global maxima of this fitness landscape (Fig. 2a).

The fitness landscape of basal gene expression resembles the fold induction landscape, with identical viable pathways, as the nodes with lower basal gene expression also show higher fold induction. All the nodes along viable pathways have lower basal gene expression than wild-type $\operatorname{TtgR}(0000)$ and the quadruple mutant is one of the mutants with lowest basal gene expression (Fig. 2b). The adaptive landscapes of maximum gene expression and $\mathrm{EC}_{50}$ show similar features to each other including a general trend of increasing magnitude from 0000 to 1111 (Fig. 2c,d). Since the global maxima for maximum gene expression is 0111 (not 1111), all pathways on the maximum gene expression landscape terminate at 0111 (Fig. 2c). Six pathways are allowed in the $\mathrm{EC}_{50}$ landscape because of the general tendency of mutations to increase $\mathrm{EC}_{50}$ regardless of mutational background (Fig. 2d). There is an interesting dependence between maximum gene expression and $\mathrm{EC}_{50}$ where nodes with high expression tended to also have high $\mathrm{EC}_{50}$ (low ligand sensitivity), indicating a likely trade off where high gene expression comes at the expense of ligand sensitivity. In other words, it may be difficult to achieve an ultrasensitive response concomitantly with a large change in gene expression. Since small deviations in activity may be permitted during evolution, we relaxed the requirement that each subsequent step through sequence space change fitness to be more like the quadruple mutant. We allowed small losses in the function of $25 \%$ between nodes and found that additional pathways are tolerated in the basal gene expression, maximum gene expression, and $\mathrm{EC}_{50}$ landscapes. No additional pathways exist in the resveratrol fold induction landscape (Supplementary Fig. 5).

Next, we delved deeper into the key epistatic interactions that shape the fitness landscapes. Epistatic interactions are classified as magnitude, sign, or reciprocal sign based on the combined effect of a pair of mutations relative to the effect of each mutant individually. Magnitude epistasis occurs when both mutations individually are beneficial or detrimental and their combined effect is greater in magnitude than the sum of their individual effects (Supplementary Fig. 6). Sign epistasis occurs when the effect of one mutation switches from beneficial to deleterious or vice versa depending on if the other mutation is present (Supplementary Fig. 6). Reciprocal sign epistasis occurs when both mutations switch effects when paired (Supplementary Fig. 6).

Two epistatic interactions, C137I-I141W and M167L-F168Y, play important roles in modulating basal gene expression and fold induction. C137I mutation makes epistatic interactions with all the other three mutations $(1100,1010$, or 1001) which are critical to control basal gene expression through sign or reciprocal sign epistasis (Fig. 2b). This is best exemplified by the interaction 

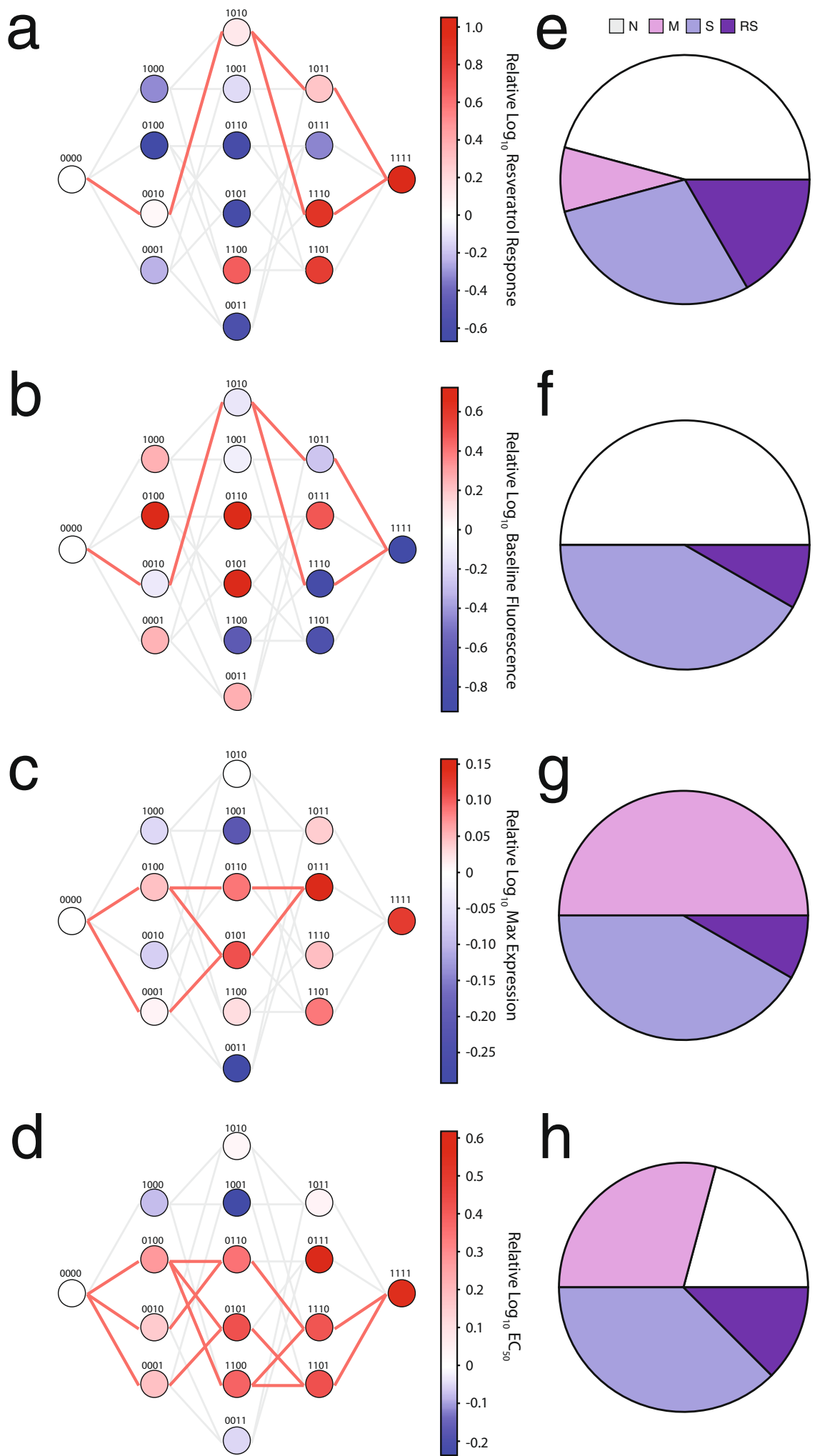

Fig. 2 Fitness landscapes for multiple functional parameters in response to induction with resveratrol. Fitness landscapes of (a) fold induction, (b) basal gene expression, (c) maximum gene expression, and (d) $\mathrm{EC}_{50}$ parameters for all $16 \mathrm{TtgR}$ variants in response to resveratrol with each variant shown as a node in the graph. Each variant is labeled with a binary string corresponding to the presence (1) or absence (0) of a mutation at position 137, 141, 167, or 168 in order. Nodes separated by a single mutation are connected by edges showing viable (bold red) and unviable paths (light gray) through sequence space. Nodes are shaded by $\log _{10}$ of the fitness parameter at $250 \mu \mathrm{M}$ resveratrol normalized to the fitness of wild-type TtgR. Number of epistatic subnetworks in the resveratrol (e) fold induction, (f) basal gene expression, (g) maximum gene expression, and (h) $\mathrm{EC}_{50}$ landscape determined by Bahadur expansion. Non-epistatic subnetworks $(\mathrm{N})$ are shown in white, magnitude epistasis $(\mathrm{M})$ in pink, sign epistasis (S) in light purple, and reciprocal sign epistasis (RS) in dark purple. 
between C137I (1000) and I141W (0100) in the basal gene expression landscape. Both 1000 and 0100 have high basal gene expression while the double mutant 1100 has low basal gene expression leading to reciprocal sign epistasis. This interaction shows mutations in the binding pocket trigger the allosteric network to create new nonspecific epistatic interactions at a distal site (in this case, the DNA-binding interface). The other double mutants that contain C137I (1010 and 1001) also have decreased basal gene expression, which is maintained through the quadruple mutant by non-epistatic (1100-1111, 1010-1111, and 1001-1111) interactions (Fig. 2b). The I141W mutation is also a key modulator of fold induction that manifests through controlling basal gene expression. Although this mutation by itself causes high basal gene expression (low fold induction) when paired with either M167L (0110) or F168Y (0101) in any combination, in the 1100 background both M167L (1110) and F168Y (1101) have low basal gene expression (high fold induction) and form a magnitude epistasis interaction to generate the phenotype of the quadruple mutant (Fig. 2b).

The M167L mutation makes a strong nonspecific epistatic pair with the F168Y mutation, creating a reciprocal sign epistasis interaction in the $\mathrm{EC}_{50}$ landscape and sign epistasis in the basal gene expression, maximum gene expression, and fold induction landscapes. In the $\mathrm{EC}_{50}$ landscape, $\mathrm{M} 167 \mathrm{~L}$ is the only node that decreases $\mathrm{EC}_{50}$ that does not contain C137I (Fig. 2d). However, this effect is masked by the addition of either C137I or I141W. The two mutations show sign epistasis in the maximum gene expression landscape in the C137I background (1000-1011) and magnitude epistasis in the I141W or C137I-I141W background, indicating that the pair behavior is dependent on the background mutations (Fig. 2c).

While a qualitative description of epistasis is easy to visualize, we wanted to also quantify the extent of and characterize the type of epistasis within all individual subnetworks and the entire 16variant system. A subnetwork is a set of four variants comprising a background variant, two single mutants and a double mutant introduced into the background variant. We used Bahadur expansion to describe all pairwise and higher order interactions (see "Methods") ${ }^{36}$. The Bahadur expansion models the activity of the landscape using a linear sum of interaction terms and coefficients. Orders of interactions (first [solo], second [pairwise], third [three way], or fourth [four way]) can be included in this sum to understand their contribution to modeling the behavior of all variants. For each subnetwork, we computed the correlation coefficient between a linear sum of first-order interaction terms and actual experimental data. In the simplest case of no epistasis, the correlation coefficient of this comparison $\left(\mathrm{R}^{2}\right)$ is close to 1 , but any deviation $\left(R^{2}<1.0\right)$ indicates the prevalence of epistasis. We applied the Bahadur expansion to quantify epistasis in all 24 subnetworks for each fitness parameter. The fitness landscapes of basal gene expression, maximum gene expression, and $\mathrm{EC}_{50}$ had unique patterns of epistatic interactions (Fig. 2e-h).

Epistasis thus has a large role in shaping the fold induction landscape between the wild type and the quadruple mutant through key interactions. The magnitude and location of the epistatic interactions are unique to their respective fitness property. Although the global expansion first-order terms explain the majority of the variance in the fold induction landscape, higher order epistatic interactions influence resveratrol fold induction by modulating interactions in secondary and tertiary subnetworks to improve the resveratrol response (Supplementary Fig. 7).

Epistasis uniquely influences the fitness landscape of each ligand. As inducer specificity changes, the fitness landscape of the same mutational intermediates will differ for each inducer. These differences may reveal alternative adaptive pathways in the fitness landscape of one inducer that circumvent functional "dead ends" in the fitness landscape of another inducer. Therefore, we examined the fitness landscape of naringenin-induced response by evaluating the same four parameters: fold induction, basal gene expression, maximum gene expression (at $2000 \mu \mathrm{M}$ ), and $\mathrm{EC}_{50}$ of all 16 variants for comparison with the fitness landscapes of resveratrol. We determined the number of viable pathways by requiring that each additional mutation must have a change in fitness that bridges wild type and the quadruple mutant to emulate the progressive change in function during evolution.

In the fold induction landscape, none of the 24 possible pathways viably connect wild type to quadruple mutant because the global minima (variant with lowest naringenin response) in the landscape is the double mutant 0110 , not the quadruple mutant (1111) (Fig. 3a). In the basal gene expression landscape, three pathways connect wild type to the quadruple mutant through the C137I (1000) mutation (Fig. 3b). Pathways emerging from 1000 pass through two double mutants, 1001 and 1100, with lower basal gene expression. The basal gene expression of 1001 is higher than 1100 , allowing 1001 to link to both triple mutants (1011 and 1101) compared to the single triple mutant from 1100 (1110). The maximum gene expression landscape contains two pathways connecting wild type to quadruple mutant (Fig. 3c). Although many nodes have lower maximum gene expression compared to the preceding node, most are not part of pathways that bridge wild type and the quadruple mutant. Two single mutants (1000 and 0100) have lower maximum gene expression than wild type, but only one is connected to a viable double mutant (0110). Both triple mutants (0111 and 1110) accessible from 0110 connect to the quadruple mutant. Like the $\mathrm{EC}_{50}$ landscape of resveratrol, the $\mathrm{EC}_{50}$ landscape of naringenin is characterized by a general increase in $\mathrm{EC}_{50}$ as mutations accumulate (Fig. 3d). There are eight possible pathways that link wild type to the quadruple mutant. Three of the four single mutants increase $\mathrm{EC}_{50}(0100,0010$, and 0001). Four of the double mutants and all the triple mutants are accessible by at least one of the preceding nodes, but not every double or triple mutant is accessible from all preceding nodes due to minor deviations in the general trend of increasing $\mathrm{EC}_{50}$. No additional mutational pathways are tolerated even when increases of up to $25 \%$ naringenin response are allowed between nodes for the naringenin fold induction landscape (Supplementary Fig. 8). Similarly to the resveratrol landscapes, the basal gene expression, maximum gene expression, and $\mathrm{EC}_{50}$ landscapes show additional pathways at this tolerance.

Closer examination of the role of individual mutations shows that $\mathrm{C} 137 \mathrm{I}$ and I141W have strong effects on multiple landscapes. $\mathrm{C} 137 \mathrm{I}$ (1000) is the only mutation that decreases $\mathrm{EC}_{50}$ relative to wild type (Fig. 3d). Two additional double mutants 1010 and 1001 further decrease $\mathrm{EC}_{50}$ but pairing C137I with I141W (1100) or C137I with both M167L and F168Y (1011) increases $\mathrm{EC}_{50}$, suggesting that these mutational combinations may mask the effect of C137I. As with the resveratrol landscapes, the I141W mutation has an important role in modulating basal gene expression and fold induction (Fig. 3a, b). Any mutant containing I141W, but not C137I has higher basal gene expression (lower folder induction) than wild type. Combining I141W and C137I results in a large decrease in basal gene expression, which further decreases upon the addition of either M167L (1110) or F168Y (1101). M167L and F168Y individually result in incremental changes in basal gene expression, maximum gene expression, and $\mathrm{EC}_{50}$ (Fig. 3b-d). However, the M167L-F168Y double mutant shows interesting context-dependent effects due to nonspecific epistasis. For example, in the fold induction landscape, the combination of M167L and F168Y is beneficial in 1000 
a

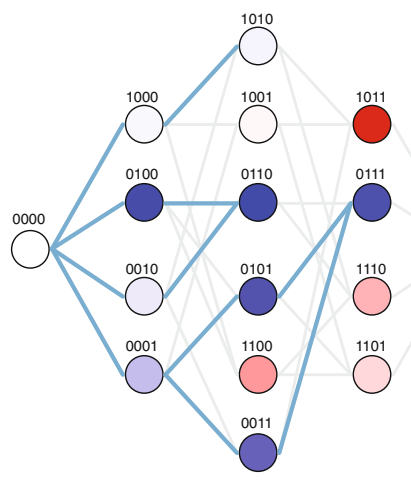

b

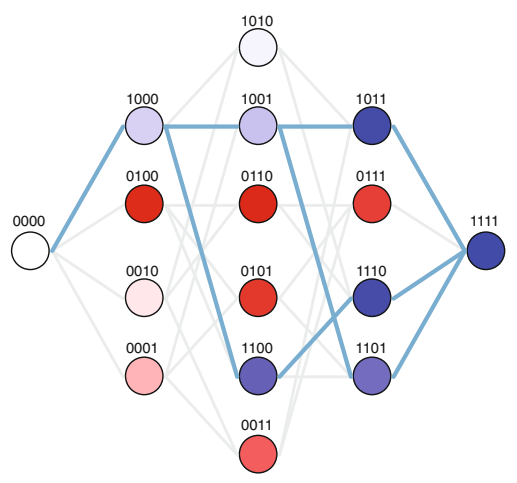

C

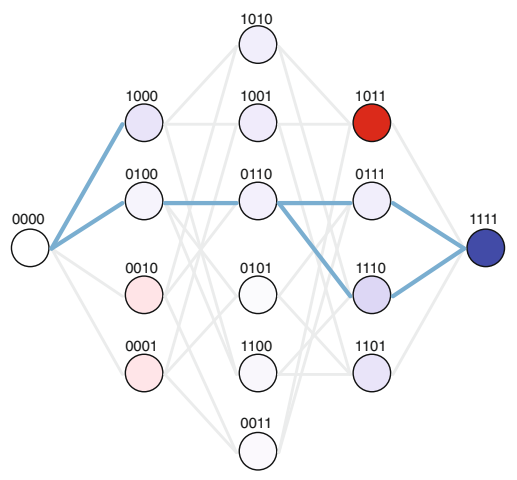

d

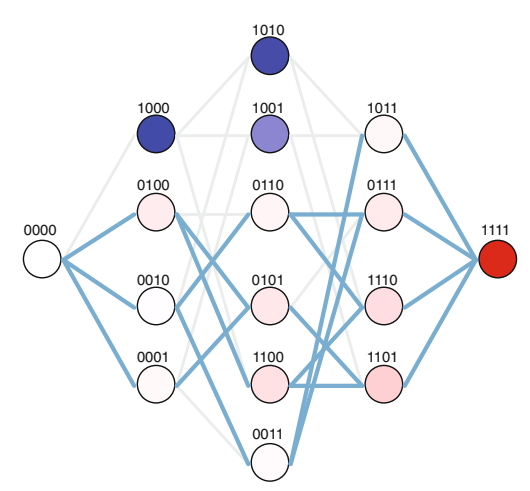

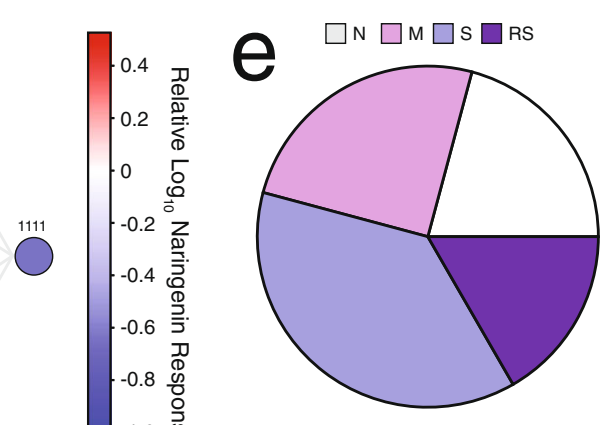

$-1.0 \stackrel{0}{\stackrel{0}{\infty}}$
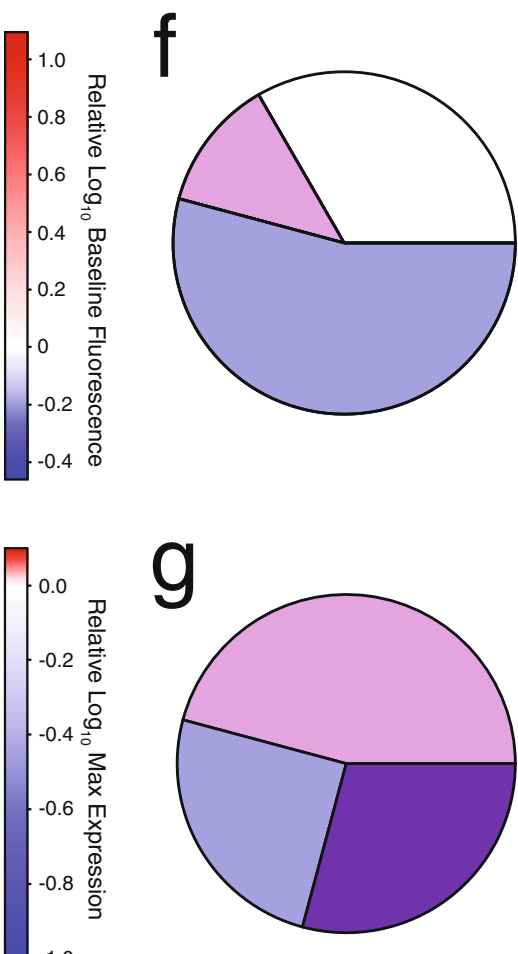

$-1.0$

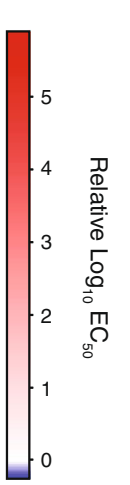

$\mathrm{h}$

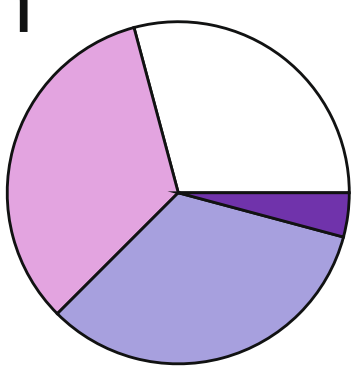

Fig. 3 Fitness landscapes for multiple functional parameters in response to induction with naringenin. Fitness landscapes of (a) fold induction, (b) basal gene expression, (c) maximum gene expression, and (d) $\mathrm{EC}_{50}$ parameters for all $16 \mathrm{TtgR}$ variants in response to naringenin with each variant shown as a node in the graph. The labeling and line colors are analogous to Fig. 2. Nodes are shaded by $\log _{10}$ of the fitness parameter at $2000 \mu \mathrm{M}$ naringenin normalized to the fitness of wild type TtgR. Number of epistatic subnetworks in the resveratrol (e) fold induction, (f) basal gene expression, (g) maximum gene expression, and $(\mathbf{h}) \mathrm{EC}_{50}$ landscape determined by Bahadur expansion. The labels of the pie charts are identical to Fig. 2. 
background but is detrimental in the 1100 background (Fig. 3a). This dependent behavior extends to all the other fitness landscapes even though the mutational background and types of epistasis change.

Epistasis shapes the fitness landscape of each function (naringenin and resveratrol response) in distinct ways. Furthermore, each functional parameter (basal gene expression, maximum gene expression, or $\mathrm{EC}_{50}$ ) is affected uniquely by the addition of multiple combinations of mutations. I141W controls high basal gene expression and strongly modulates fold induction regardless of ligand. In contrast, C137I is more context-dependent; it is responsible for low $\mathrm{EC}_{50}$ values solo or in combination with either M167L or F168Y in the naringenin landscape but is strongly influenced by $\mathrm{M} 167 \mathrm{~L}$ in the resveratrol $\mathrm{EC}_{50}$ landscape. Some epistatic pairs are consistent between the resveratrol and naringenin landscapes. The C137I + I141W pair strongly affects basal gene expression and fold induction for both ligands. The M167L + F168Y pair has unique behavior in all fitness landscapes that is dependent on the mutation background into which they are introduced. However, the pair's effect on the wild type background is stronger in resveratrol compared to naringenin for all parameters. The patterns of epistasis in the naringenin subnetworks are unique to their respective functional parameter (Fig. 3e-h). Furthermore, the same set of mutations that create epistatic interactions giving rise to high resveratrol response forge ligand-specific epistatic patterns in the fold induction, basal gene expression, maximum gene expression, and $\mathrm{EC}_{50}$ landscapes (Supplementary Fig. 9).

Crystal structure reveals molecular basis of specificity of quadruple mutant. To understand the structural basis of TtgRligand interactions, we solved high-resolution crystal structures of quadruple mutant (resveratrol-bound and apo) and wild-type TtgR (resveratrol-bound) at a resolution of $1.9 \AA$ or better (Supplementary Table 3 ). TtgR is a compact, dimeric, all-helical transcription factor with a large cavity between five angled helices forming the ligand-binding pocket (Supplementary Fig. 10a, b). The quadruple mutant bound to resveratrol (PDB: 7KD8) is structurally very similar to the wild type with an all-atom RMSD of $1.2 \AA$ over the entire structure. The DNA-binding domains of the resveratrol-bound quadruple mutant and the resveratrolbound wild type are extremely similar with an all-atom RMSD of $1.0 \AA$ (Supplementary Fig. 11). The four mutations do not substantially change the volume of the pocket $\left(215 \AA^{3}\right.$ in wild type compared to $234 \AA^{3}$ in the quadruple mutant) or the surface area of the pocket ( $184 \AA$ in wild type compared to $186 \AA$ in the quadruple mutant) (Supplementary Fig. 12). The position and orientation of resveratrol in the wild-type TtgR structure (PDB: $7 \mathrm{~K} 1 \mathrm{C})$ resembles the position and orientation of naringenin in a previously solved co-crystal structure of TtgR (PDB: $2 \mathrm{UXU})^{28}$. In both structures, the ligands bind in a vertical mode such that the plane of the molecule is roughly perpendicular to DNA (Supplementary Fig. 10c). In wild-type TtgR, the four mutated positions (C137, I141, M167, and F168) are located approximately in the center of the binding pocket and make nonspecific van der Waals interactions with resveratrol (Fig. 4a, upper panel). Other neighboring residues N110, D172 and H114 make specific hydrogen bonds that stabilize resveratrol in the vertical orientation (Fig. 4a, lower panel). Although both naringenin and resveratrol bind in the vertical orientation, only N110 is able to make a hydrogen bond with both naringenin and resveratrol ${ }^{28}$. The ability of wild-type TtgR to bind multiple ligands likely arises from the nonspecific interactions made by the nonpolar amino acids in the binding pocket.
The structure of the quadruple mutant reveals the role of individual residues in ligand specificity. I141W, a mutation critical for resveratrol specificity, creates a large steric barrier that alters the shape of the pocket and obstructs the vertical binding orientation of ligands (Fig. 4b, upper panel). Resveratrol is accommodated in the binding pocket in a horizontal binding orientation almost parallel to the plane of the tryptophan. Unlike I141W which plays a clear steric role, the other three mutations (C137I, M167L and F168Y) have a more subtle effect in reshaping the binding pocket through nonpolar interactions. C137I mutation creates a protrusion in the binding pocket that increases shape complementarity to resveratrol (Supplementary Fig. 13a). M167L is buried between the residues in the binding pocket and the dimerization helix and may play a role in positioning the I141W tryptophan to stabilize its horizontal orientation through van der Waals interactions (Supplementary Fig. 13b). F168Y allows the formation of multiple hydrogen bonds with nearby water molecules and may serve to stabilize the structure (Supplementary Fig. 13b). A different hydrogen bonding network consisting of D71, R75, and E78 make hydrogen bonds with the resveratrol molecules in chain A (Supplementary Fig. 13c) and D71, E78, D172, and a nearby water molecule make a hydrogen bond with the single resveratrol molecule in chain B (Fig. $4 \mathrm{~b}$, lower panel).

Although resveratrol and naringenin share similar chemical backbones, naringenin is bulkier than resveratrol due to the fused carbon rings of the chromanone. This reduces the shape complementarity of naringenin to the redesigned binding pocket despite the similarity in the volume of the quadruple mutant and wild-type binding pockets (Supplementary Figs. 12, 14). The

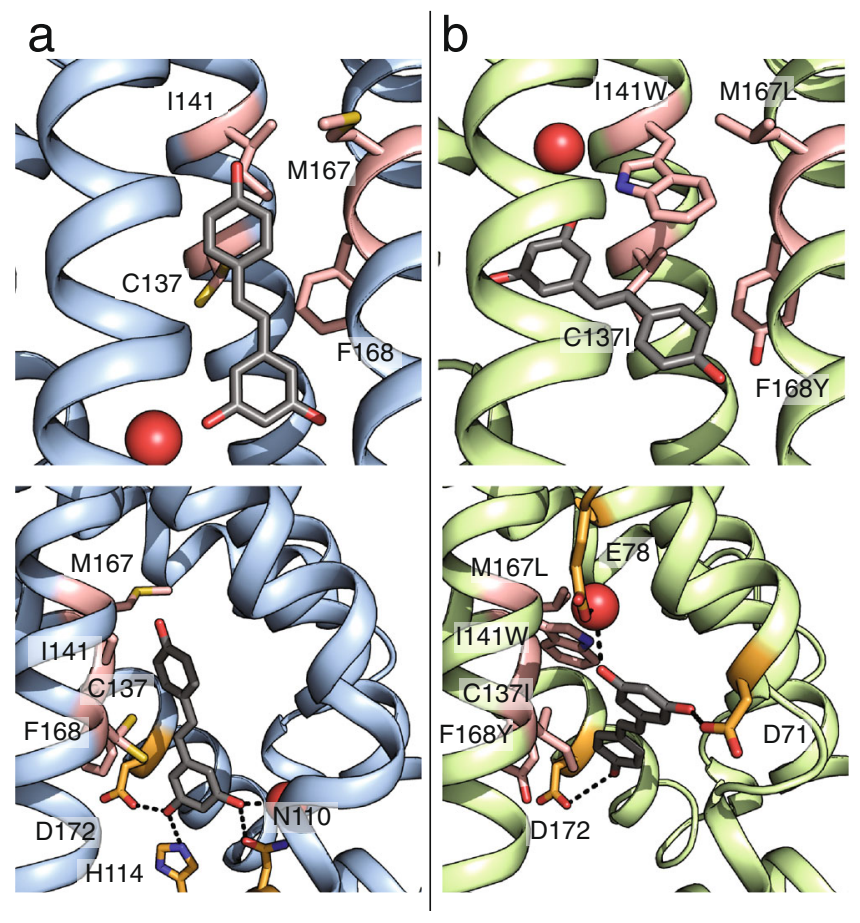

Fig. 4 Structural basis for ligand specificity. Wild-type $T \operatorname{tg} R$ and quadruple mutant are shown in blue and green ribbons, respectively. Positions 137, 141, 167, and 168 are colored in pink. Resveratrol is shown as gray sticks. Water molecules are shown as red spheres. a Binding pocket of resveratrol-bound wild-type TtgR (PDB ID: 7K1C) (upper panel) with residues making hydrogen bonds to resveratrol highlighted in orange (lower panel). b Binding pocket of resveratrol-bound quadruple mutant TtgR (PDB ID: 7KD8) (upper panel) with residues making hydrogen bonds to resveratrol highlighted in orange (lower panel). 
4-hydroxyphenyl moiety and the carbonyl group of the 4-chromanone backbone of naringenin could create steric clashes with residues lining the wall of the pocket and cause the ligand to sample less space in the pocket compared to resveratrol, which provides a reasonable structural basis for ligand specificity.

The new binding mode of the quadruple mutant was not predicted in the original design scheme. We seeded the input structures for the Rosetta design with resveratrol docked in the vertical orientation to mimic the binding mode of the wild-type structure. The design process is only able to make minor alterations to the position and angle of the ligand in the binding pocket (Supplementary Fig. 15). However, Rosetta was able to identify a subset of positions that, when mutated, could confer resveratrol specificity.

The structural basis of ligand specificity relies on the I141W substitution to create a steric barrier to prevent binding in the vertical orientation, which is observed in wild-type TtgR for multiple ligands. In the novel horizontal mode, other ligands may be occluded from the pocket through steric clashes with wild-type residues in the pocket. The epistatic interactions observed in the fitness landscapes for naringenin and resveratrol can be rationalized through examination of the structure. The C137II141W pair increases shape complementarity to resveratrol while M167L-F186Y contact the dimerization helix and potentially affect the positioning of nearby residues that interact with the ligand. The altered binding mode establishes that allostery is robust to major changes in the binding mode in TtgR.

Relationship between biophysical affinity and biological response. Ligand response of an aTF is a complex combination of both biophysical interactions and allostery. Mutations that affect aTF fold induction can do so by altering ligand affinity, DNA affinity, or the allosteric signal upon ligand binding. Since all four mutations are localized to the binding pocket, the observed changes in fold induction of TtgR are likely due to altered binding affinity to ligand, the transmission of allosteric signal, or both. To understand the relationship between biophysical affinity and biological response, we compared changes in ligand affinity $\left(\mathrm{K}_{\mathrm{d}}\right)$ to changes in ligand sensitivity $\left(\mathrm{EC}_{50}\right)$ for both naringenin and resveratrol. We chose mutants in the 0000-1000-0100-1100 subnetwork because it is important for the high resveratrol response in the quadruple mutant. Further, this network shows a strong manifestation of nonspecific epistasis through reciprocal sign change and is therefore a good model to understand the relationship between biophysical affinity and biological response. We estimated ligand affinity using isothermal titration calorimetry (ITC) of purified proteins and ligand sensitivity from dose-response curves. Ligand sensitivity is derived from reporter expression and is thus a combination of both allostery and affinity.

Affinity and sensitivity of resveratrol for different variants are generally concordant for resveratrol, with the exception of 1100 (Fig. 5a). We note that the ITC and dose-response curves for some variants did not plateau due to poor ligand solubility at high concentrations resulting in imprecise estimates of $\mathrm{K}_{\mathrm{d}}$ and $\mathrm{EC}_{50}$. Nonetheless, qualitative comparisons can be made to gain useful insight. For instance, comparison of ITC profiles of 0000 and 1111 for resveratrol shows weaker binding for 1111 even though the precise $\mathrm{K}_{\mathrm{d}}$ may be difficult to measure. Similarly, dose-response curves show weaker $\mathrm{EC}_{50}$ for 1111 than 0000 even though it is not fully saturated. The C137I mutation appears to be largely responsible for the affinity in 1100 , but the I141W mutation causes the increase in sensitivity. In general, as mutations accumulate from wild type, the affinity and sensitivity generally decrease, suggesting a decreased ability to undergo allosteric changes is likely due to weaker binding (Fig. 5a). The discordance between affinity and sensitivity is much greater for naringenin than resveratrol. In the case of naringenin, no relationship was evident between affinity and sensitivity across the subnetwork (Fig. 5b). Although the quadruple mutant has higher resveratrol fold induction than wild type, its affinity and sensitivity for resveratrol are lower than that of wild type (Figs. 1, 5a). In essence, these examples illustrate the complex relationship between local interactions (specific epistasis) and their global effects in allosteric proteins.

The 0000-1000-0100-1100 subnetwork displays a unique, ligand-specific pattern of specific epistasis for biophysical and biological parameters. The mutations we introduced into TtgR suggest an effect on allostery changes in $\mathrm{EC}_{50}$ as the complexities of function may not be simply explained by changes in biophysical affinity. These measurements also suggest that by optimizing a particular protein function (fold induction), other
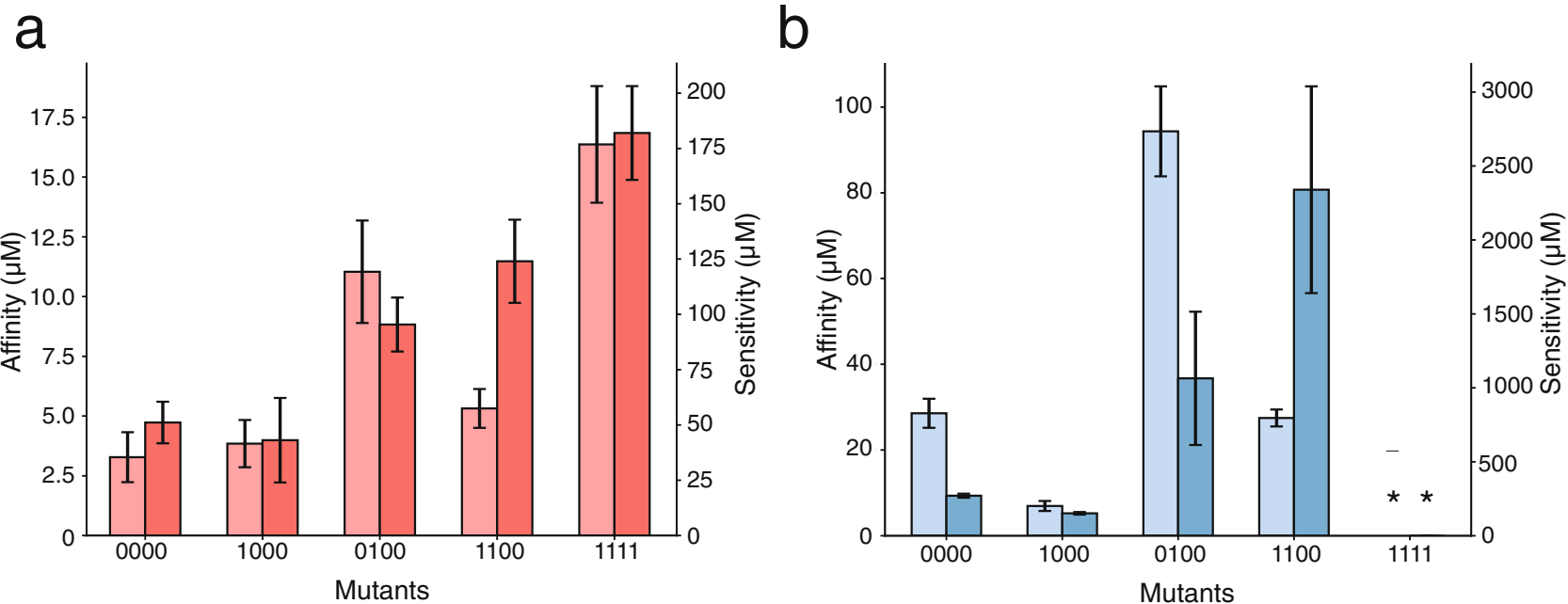

Fig. 5 Comparison of biophysical and biological properties of TtgR variants. Ligand affinity (light bar) and $\mathrm{EC}_{50}$ sensitivity (dark bar) for resveratrol (a) and naringenin (b) are shown for TtgR variants $0000,1000,0100,1100$, and 1111 . Ligand affinity was estimated by isothermal calorimetry and $\mathrm{EC}_{50}$ sensitivity from fitting dose-response curves to the Hill equation. $\mathrm{EC}_{50}$ values and error are calculated based on fitting to triplicate dose-response curves. ITC values and error are generated from a one-site binding model; error bars denote the error of the fit (see methods). The values shown are likely to underestimate the actual affinity and sensitivity values. 
parameters (sensitivity or affinity) may not necessarily stay at fitness maxima as the 1111 mutant shows poor sensitivity (high $\mathrm{EC}_{50}$ ) to both ligands.

\section{Discussion}

In this study, we describe the pervasive effects of epistasis on ligand specificity in a simple allosteric transcription factor by examining fold induction, basal gene expression, maximum gene expression, and $\mathrm{EC}_{50}$ of two ligands across multiple mutants. By leveraging computational protein design, we engineered four mutations into $\mathrm{TtgR}$, a transcription factor that can normally bind to both resveratrol and naringenin, to only bind to resveratrol. By characterizing the functional response to both resveratrol and naringenin across all combinations of mutations, we show that the extent of epistasis between mutations affecting multiple protein functions is specific for each ligand. For instance, $50 \%$ of subnetworks meet the criteria for epistasis for resveratrol fold induction while $83 \%$ of subnetworks are epistatic for naringenin fold induction. However, the fitness landscapes of both ligands are shaped by common critical pairs of epistatic interactions (C137I and I141W or M167L and F168Y), though their behavior may be different depending on the functional parameter. The biological effects of these mutations are further validated by the crystal structures. The four mutations localize to one face of the binding pocket, making nonpolar interactions with the ligand. C137I and I141W increase shape complementarity of the pocket for resveratrol, but only in an alternative horizontal binding pose. The four mutations that confer ligand specificity decrease both affinity and sensitivity suggesting that the changes in sensitivity could be a consequence of lower affinity and not necessarily a purely allosteric effect.

Our study used a constrained set of mutations chosen through in silico selection as opposed to the selection of random mutations through natural evolution. An evolutionary process may have selected a different set of mutations to confer the same functional outcome, leading to the presence of a different pattern of epistasis for either naringenin or resveratrol response. Often in natural evolution, mutations that are distal to the site of interest have a profound effect on protein function ${ }^{8,21}$. These background mutations complicate any examination of key mutations within the targeted area of the protein and their influence on protein function. By utilizing a combination of computational design and high-throughput screening, we targeted mutations to a discrete set of ligand-interacting positions within the binding pocket. Our approach enabled us to examine the propensity of epistasis in a constrained setting where mutations are limited to those that interact directly with the ligand, enabling the examination of the intersection of mutation, biophysical epistasis, and biological epistasis.

Our results highlight the dependence of epistasis on protein function and the prevalence of distinctive adaptive landscapes for multiple functions within the same set of mutations. This process highlights the functional tradeoffs that occur during an evolutionary process and raises the implication that proteins with multiple functions may readily traverse nonoptimal sequence space through varying selective pressures. These landscapes can thus become interconnected by changing selection pressures between different protein functions. On an evolutionary scale, simultaneously changing protein sequence and selection pressure may enable improbable trajectories by bypassing epistatic barriers to reach previously inaccessible mutational states. In our case, higher order epistasis which prevents access to the quadruple mutant in the naringenin fold induction landscape, could be bypassed by toggling between naringenin and resveratrol selection pressures. The evolution of allosteric proteins is inherently dependent on epistasis and the interactions arising between mutations in these proteins uniquely affects multiple adaptive landscapes.

\section{Methods}

Computational design. Protein modeling and design was performed with Rosetta version 3.5 (2015.19.57819) ${ }^{35,37}$. Python and shell scripts for generating input from Rosetta and analyzing from Rosetta are available at: https:/github.com/raman-lab/ biosensor_design

The high-resolution TtgR structure co-crystalized with tetracycline was selected as the starting point for computational design (PDB: $2 \mathrm{UXH})^{28}$. The structure was prepared for use in Rosetta by performing an all-atom, coordinate-constrained relaxation ${ }^{38}$

Rosetta/main/source/bin/idealize_jd2.linuxgccrelease -database Rosetta/main/ database/ -in::file::fullatom -s 2UXH.pdb -extra_res_fa LG.params -no_optH false -flip_HNQ

Rosetta/main/source/bin/relax.linuxgccrelease -database Rosetta/main/ database/ -relax::sequence_file always_constrained_relax_script -constrain_relax_to_native_coords -relax::coord_cst_width 0.25 -relax:::coord_cst_stdev 0.25 -s 2UXH_idealized.pdb -in::file::native 2UXH_idealized.pdb -extra_res_fa LG.params -in::file::fullatom -no_optH false -flip_HNQ

Rosetta/main/source/scripts/python/public/molfile_to_params.py -n resveratrol.params -p resveratrol.pdb

The RosettaScripts protocol used to design the ligand-binding pocket of each starting TtgR-resveratrol complex was based on enzyme design protocols ${ }^{32,39}$.

Rosetta/main/source/bin/rosetta_scripts.linuxgccrelease -database Rosetta/ main/database/ -parser::protocol enzdes.xml -in::file::s 2UXH_resvertrol.pdb -extra_res_fa resv.params -use_input_sc -packing:linmem_ig 10 -ex1-ex2 -run:preserve_header -enzdes_out -enzdes:bb_min_allowed_dev 0.2 -enzdes:loop_bb_min_allowed_dev 0.5 -enzdes:minimize_ligand_torsions 15 -parser::script_vars ligchain $=\bar{X}$ resfile $=$ TtgR.resfile -out::pdb - nstruct 10

The TtgR.resfile is a plain text file containing the amino acid position numbers that were able to be mutated during design, and these were positions 137, 141, 167, $168,171,172,175$, and 176. We used UW-Madison's Center for High Throughput Computing computer cluster to perform 320,000 different design simulations. The resulting designed structures were curated to yield the set of sequences that we synthesized to isolate resveratrol-specific TtgR variants.

We selected computational designs for synthesis by first removing designs that were repetitive and then removing designs that were energetically unfavorable. The criteria for unfavorable energies were selected empirically based on the distribution of energies for all designs to yield approximately $10^{4}$ sequences for synthesis. Specifically, on each unique design, $\Delta \Delta \mathrm{G}$ stability calculations were performed on designed residues to ensure the number of destabilizing changes was limited. If the mutation destabilized the TtgR-resveratrol complex by 0.5 Rosetta Energy Units (REU), the residue was reverted to its wild-type identity. After this, non-unique designs were again removed. The unique designs were filtered using distance from the median absolute deviation of several salient Rosetta scoring metrics including total ligand binding energy, hydrogen bond energy, Leonard-Jones repulsive energy, solvation energy, and total score, which is a weighted, linear combination of all score terms in the energy function ${ }^{34}$. Designs that passed this filter were synthesized for library screening.//biosensor_design/fas_from_pdb_stdout.py *.pdb > TtgR_resveratrol_all_designs.fasta./biosensor_design/uniquify_fas.py TtgR_resveratrol_all_designs.fasta $>$ TtgR_resveratrol_unique_designs.fasta./ ddg_monomer.static.linuxgccrelease -database./database @ddg_flags -in:file:s design_pdb.pdb -ddg::mut_file list_of_positions_to_calc_ddg.mutfile -ddg::iterations 50./gen_enzdes_cutoffs.py concatentated_design_score_file.sc -c median_abolute_deviation_cutoffs.txt -o designs_passing_filter.sc

The median absolute deviation cutoffs used were:

total_score $<+1$ MAD

fa_rep $<+3$ MAD

hbond_sc $<+3$ MAD

tot_burunsat_pm $<+3 \mathrm{MAD}$

$\%$ (LIG)s_fa_rep $<+3 \mathrm{SD}$

$\%($ LIG)s_hbond_sc $<+3$ MAD

$\%$ (LIG)s_burunsat_pm $<2.5$ ABS

$\%($ LIG)s_total_score $<-1$ MAD

Library synthesis. The sfGFP reporter plasmid was constructed using a backbone containing the ColE1 origin and a kanamycin resistance gene. The TtgR operator sequence was modified to contain canonical $-10\left(5^{\prime}\right.$-TATAAT- $\left.3^{\prime}\right)$ and $-35\left(5^{\prime}\right.$ TTGACA-3 $3^{\prime}$ ) elements in the promoter. A strong RBS (g10) was chosen for high sfGFP expression ${ }^{40}$. The TtgR operator-RBS sequence was constructed via sequential PCR reactions with overlapping primers containing homology to the pColE1 backbone $5^{\prime}$ of sfGFP (Supplementary Table 1). The plasmid was annealed using isothermal assembly using $0.16 \mathrm{pmol}$ of backbone and $0.43 \mathrm{pmol}$ of promoter ${ }^{41}$. DH10B cells (NEB) were transformed with the pColE1 reporter plasmid and plated on LB-kanamycin agar $(50 \mu \mathrm{g} / \mathrm{mL})$. A colony was selected and grown in LB-kanamycin media $(50 \mu \mathrm{g} / \mathrm{mL})$ shaking for $16 \mathrm{~h}$ at $37^{\circ} \mathrm{C}$. An aliquot of 
the culture was stored at $-80^{\circ} \mathrm{C}$ in $25 \%$ glycerol. Plasmids were isolated using a DNA miniprep kit (Omega BioTek) according to the manufacturer's protocol. The insertion of TtgR operator sequence was confirmed via Sanger sequencing.

The TtgR expression plasmid used the SC101 origin and a spectinomycin resistance gene. The constitutive promoter-RBS combination apFAB61-BBa_J61132 and the TtgR gene were amplified via KAPA HiFi PCR mix (Roche) using primers with homology to the pSC101 backbone ${ }^{42}$. The TtgR-pSC101 construct was generated using isothermal assembly $(0.046 \mathrm{pmol}$ backbone and $0.24 \mathrm{pmol}$ TtgR) and DH10B cells were transformed with the TtgR-pSC101 construct. A colony was selected and grown in LB-spectinomycin media $(50 \mu \mathrm{g} / \mathrm{mL})$ shaking for $16 \mathrm{~h}$ at $37^{\circ} \mathrm{C}$. An aliquot was stored at $-80^{\circ} \mathrm{C}$ and plasmids were isolated and verified as described previously.

Rosetta-designed sequences were synthesized as exact oligos (Twist Biosciences). Oligos were converted to double-strand DNA using qPCR and purified on a spin column (EZNA Cycle Pure kit from Omega BioTek). The pSC101 backbone was amplified with two separate primer pairs encoding BsaI cut sites that matched the insertion location of the oligos on the TtgR gene. The amplified backbone was treated with Dpn1 for $16 \mathrm{~h}$ at $37^{\circ} \mathrm{C}$ (NEB) followed by a purification using a spin column. The backbone was treated with BsaI (NEB) for $2.5 \mathrm{~h}$ at $37^{\circ} \mathrm{C}$ followed by purification using a spin column. The digested backbone was treated with Antarctic phosphatase (NEB) for $1 \mathrm{~h}$ at $37^{\circ} \mathrm{C}$ followed by purification using a spin column. A golden gate reaction (NEB) was performed using $0.12 \mathrm{pmol}$ backbone and $0.89 \mathrm{pmol}$ library oligo in roughly a $1: 7$ molar ratio and incubating for $30 \mathrm{cycles}$ of $37^{\circ} \mathrm{C}$ for $5 \mathrm{~min}$ and $16^{\circ} \mathrm{C}$ for $5 \mathrm{~min}$ followed by $60{ }^{\circ} \mathrm{C}$ for $5 \mathrm{~min}$. A control reaction was made using just the pSC101 backbone with no Rosetta oligos added. The golden gate reactions were dialyzed using semipermeable membranes (Millipore) for $1 \mathrm{~h}$ at $25^{\circ} \mathrm{C}$ against $\mathrm{dH}_{2} \mathrm{O} .25 \mu \mathrm{L}$ of $\mathrm{C} 3020$ cells (NEB) were transformed with $2 \mu \mathrm{L}$ of the dialyzed golden gate mixture via electroporation. Cells recovered for $1 \mathrm{~h}$ in SOC media shaking at $37^{\circ} \mathrm{C}$ and were diluted 5X with LB. Dilutions of 100X, 500X, and 1000X were plated to calculate transformation efficiency relative to the control. A transformation was considered successful when $\mathrm{CFU} / \mathrm{mL}$ of the Golden Gate reactions exceeded CFU/mL of control reactions by a factor of 10 or more. Cells grew for $6 \mathrm{~h}$ post-transformation before the culture was diluted 50X and grown overnight shaking at $37^{\circ} \mathrm{C}$ for $16 \mathrm{~h}$. Plasmids of the library were harvested using a DNA miniprep kit and stored at $-20^{\circ} \mathrm{C}$.

An aliquot of the pColE1 frozen stock was streaked on a LB-kanamycin agar plate and grown for $16 \mathrm{~h}$ at $37^{\circ} \mathrm{C}$. A single colony was selected and grown in LBkanamycin media shaking for $16 \mathrm{~h}$ at $37^{\circ} \mathrm{C}$. The culture was diluted $50 \mathrm{X}$ and grown at $37^{\circ} \mathrm{C}$ to an $\mathrm{OD}_{600}$ of 0.6 . Cells were placed on ice and $5 \mathrm{~mL}$ aliquots were centrifuged at $5,500 \mathrm{~g}$ for $5 \mathrm{~min}$ at $4^{\circ} \mathrm{C}$. Pellets were resuspended, washed with ice cold $\mathrm{dH} 2 \mathrm{O}$, and spun at $5,500 \mathrm{~g}$ twice. The cells were resuspended in $20 \mu \mathrm{L}$ of water to create electrocompetent DH10B containing the pColE1 plasmid. DH10B E.coli containing the pColE1 reporter plasmid were transformed with the initial Rosetta library in pSC101 via electroporation. The transformed cells were recovered for $1 \mathrm{~h}$ shaking at $37^{\circ} \mathrm{C}$ before dilutions were plated on LB-kanamycin/spectinomycin agar plates $(50 \mu \mathrm{g} / \mathrm{mL}$ each) to calculate transformation efficiency. The remaining cells were diluted 5X with LB- kanamycin/spectinomycin media and grown shaking at $37^{\circ} \mathrm{C}$ for $16 \mathrm{~h}$. A frozen stock was made with $25 \%$ glycerol.

Fifty microliters of aliquots of the cotransformed Rosetta libraries were thawed on ice and inoculated into $5 \mathrm{~mL}$ of LB-kanamycin/spectinomycin and grown shaking at $37^{\circ} \mathrm{C}$ to an $\mathrm{OD}_{600}$ of 0.2 . Wild-type cotransformed $\operatorname{Ttg} R$ sensor + reporter was also inoculated as a reference. These were then split into $41 \mathrm{~mL}$ aliquots and inoculated with either $500 \mu \mathrm{M}$ naringenin (DMSO), $95 \mu \mathrm{M}$ resveratrol (ethanol), DMSO, ethanol and grown for $14 \mathrm{~h}$ at $37^{\circ} \mathrm{C}$ shaking. Cells were diluted 50X in ice cold PBS $\left(137 \mathrm{mM} \mathrm{NaCl}, 2.7 \mathrm{mM} \mathrm{KCl}, 10 \mathrm{mM} \mathrm{Na}_{2} \mathrm{HPO}_{4}, 1.8 \mathrm{mM} \mathrm{KH}_{2} \mathrm{PO}_{4}\right.$ ) and stored on ice prior to sorting.

Sorting was conducted using a Sony SH800 cell sorter. Cells were excited by a $488 \mathrm{~nm}$ laser and GFP fluorescence was captured through a 525/50 filter. Gain settings were adjusted such that all cells fell between $10^{2}$ and $10^{6}$ RFU. 100,000 event measurements of all libraries, induced and repressed, were taken to draw gates according to population percentage.

Sorting followed an induced-repressed schema; the first library sort consists of taking 500,000 cells of median 50\% of fluorescence from the nontreated distribution (Supplementary Fig. 16). This sort isolates cells that contain TtgR variants capable of repressing GFP expression. Cells were sorted into $2 \mathrm{~mL}$ of $\mathrm{LB}$. $\mathrm{LB}$ as added to a final volume of $5 \mathrm{~mL}$ and incubated for $1 \mathrm{~h}$ at $37^{\circ} \mathrm{C}$ shaking. Kanamycin and spectinomycin were added after $1 \mathrm{~h}$ to a final concentration of $50 \mu \mathrm{g} / \mathrm{mL}$ each from $1 \mathrm{mg} / \mathrm{mL}$ stocks. These grew to an $\mathrm{OD}_{600}$ of 0.2 before frozen stocks were made in $25 \%$ glycerol. A small aliquot was stored as a frozen stock at $-80^{\circ} \mathrm{C}$ in $25 \%$ glycerol. The remaining culture was induced with naringenin, resveratrol, DMSO, or ethanol at an $\mathrm{OD}_{600}$ of 0.2 .

The next sort consisted of isolating 100,000 cells in the top 5\% of fluorescence from the resveratrol-induced library (Supplementary Fig. 16). This subpopulation was grown as described previously and induced with $95 \mu \mathrm{M}$ resveratrol at an $\mathrm{OD}_{600}$ of 0.2 . The final sort consisted of isolating 500,000 cells from the bottom $60 \%$ of the nontreated fluorescence distribution. The sorted cells were incubated at $37^{\circ} \mathrm{C}$ until the culture reached an $\mathrm{OD}_{600}$ of 0.2 . A frozen stock was stored at $-80^{\circ} \mathrm{C}$ in $25 \%$ glycerol.

Aliquots of the sorted library, wild-type TtgR cotransformed with the reporter plasmid, and a GFP-positive control were thawed on ice. $50 \mu \mathrm{L}$ of the library was plated on LB-kanamycin/spectinomycin and incubated at $37^{\circ} \mathrm{C}$ for $16 \mathrm{~h}$. The GFP control aliquot was streaked on LB-kanamycin and the wild-type TtgR aliquot was streaked on LB-kanamycin/spectinomycin and incubated in the same fashion. Colonies were selected from each plate and inoculated into $150 \mu \mathrm{L}$ of $\mathrm{LB}$ in a 96 well plate. The colonies were incubated at $37^{\circ} \mathrm{C}$ shaking in a SBT1500- $\mathrm{H}$ microplate shaker (Southwest Science) and grew to saturation (approximately $8 \mathrm{~h}$ ). The cultures were diluted 15X into fresh LB with either $1000 \mu \mathrm{M}$ naringenin or $100 \mu \mathrm{M}$ resveratrol and incubated in a Synergy HTX plate reader (BioTek) for $16 \mathrm{~h}$ at $37^{\circ} \mathrm{C}$. The performance of each colony was measured using the ratio of fluorescence to optical density $\left(\mathrm{RFU} / \mathrm{OD}_{600}\right)$. The ratio of this measurement in the presence and absence of ligand defined the response to each ligand. Successful colonies had higher response for resveratrol than for naringenin. These colonies were sequenced using Sanger sequencing.

Testing of combinatorial mutants. The 14 mutational intermediates were generated using eight primers specifically encoding combinations of either $137+141$ or $167+168$. The resulting oligos were inserted into the TtgR-pSC101 plasmid using isothermal assembly using .042pmol of backbone and $0.8 \mathrm{pmol}$ TtgR. DH10B E.coli cells (NEB) were transformed with the resulting reaction via electroporation. Colonies were selected and sequenced to verify the correct mutations were present. The correct colonies were inoculated into LB-spectinomycin and incubated at $37^{\circ} \mathrm{C}$ for $16 \mathrm{~h}$. An aliquot was stored at $-80^{\circ} \mathrm{C}$ in $25 \%$ glycerol and plasmids were harvested from the remaining culture. DH10B cells were cotransformed with the 14 TtgR-pSC101 plasmids and the pColE1 reporter plasmid. These were grown for $16 \mathrm{~h}$ shaking at $37^{\circ} \mathrm{C}$ in LB-kanamycin/spectinomycin media and frozen in $25 \%$ glycerol at $-80^{\circ} \mathrm{C}$.

A $250 \mathrm{mM}$ stock of naringenin was made in DMSO and a $100 \mathrm{mM}$ stock of resveratrol was made in ethanol. The TtgR-pSC101/pColE1 frozen stocks were struck out onto LB-kanamycin/spectinomycin plates. Colonies were selected and inoculated into $150 \mathrm{uL} \mathrm{LB}$ in a 96 -well plate. These grew in a microplate shaker to saturation (approximately $8 \mathrm{~h}$ ) at $37^{\circ} \mathrm{C}$. The cultures were diluted $15 \mathrm{X}$ into fresh LB-kanamycin/spectinomycin in a 96-well plate with varying concentrations of either naringenin $(0,10,25,50,75,100,250,500,750,1000,1500,2000 \mu \mathrm{M})$ or resveratrol $(0,2.5,5,7.5,10,25,50,75,100,150,200,250 \mu \mathrm{M})$. The concentration series for each ligand differ due to solubility limits in aqueous solutions. A series of naringenin and resveratrol stock concentrations were made such that a $50 \mathrm{X}$ or a $100 \mathrm{X}$ dilution, respectively, would yield the desired concentrations in the assay. Most variants were assayed with three biological replicates. Variants whose standard deviation was greater than $10 \%$ of the mean fluorescence $(1010,1001$, 1110, and 1101 for naringenin and 1001, 1000, 0001, and 0011 for resveratrol) were assayed with six replicates. The assay was incubated in the microplate shaker for $14 \mathrm{~h}$ at $37^{\circ} \mathrm{C}$ shaking. Cells containing wild-type TtgR-pSC101 with the pColE1 reporter and cells containing pColE1 reporter alone served as controls and were included on every plate. A set of six biological replicates of a sfGFP positive control were induced with both sets of ligands and concentrations.

Cells were diluted 50X in ice-cold PBS. Fluorescence measurements were conducted on a LSR-Fortessa system (BD Biosciences) in the FACSDiva V8.0 software using a $488 \mathrm{~nm}$ laser for excitation and a 530/30 filter for fluorescence emission. Using gates on FSC-H vs FSC-A, 100,000 events were gathered per well (Supplementary Fig. 16). To account for changes in fluorescence that are independent of $\operatorname{TtgR}$ function, raw fluorescence values were normalized by fold changes in sfGFP fluorescence in the positive control $(N=6)$. The median values of the fluorescence distributions were used as the basis for fold induction calculations. Fold induction as calculated by obtaining the ratio of induced average median fluorescence to baseline average median fluorescence. In Fig. 1, fold induction values were calculated by obtaining the ratio of each biological replicate prior to averaging the ratios.

$$
\text { fold induction }=\frac{F_{\text {max }}}{F_{\text {baseline }}}
$$

Quantifying epistasis. The mean and standard deviation of each concentration of ligand for each combinatorial mutant were used to calculate a fit using the Hill equation as a function of ligand concentration $(x)^{43}$ :

$$
f\left(x, n, E C_{50}\right)=F_{\text {baseline }}+\left(\left(F_{\text {max }}-F_{\text {baseline }}\right) *\left(\frac{x^{n}}{E C_{50}{ }^{n}+x^{n}}\right)\right)
$$

TtgR function was defined as the maximum fold induction of the system, which is the ratio of the median fluorescence at the highest ligand concentration and the median fluorescence at $0 \mu \mathrm{M}$ ligand (Eq. 1). The Python 2.7 function curve_fit() from the Scipy module was used to fit the dose-response curves to the Hill equation (Supplementary Figs. 17, 18) ${ }^{44}$. This function provides both fit parameters and error as a covariance matrix as output. Basal gene expression was the fluorescence at $0 \mu \mathrm{M}$ ligand. Maximum gene expression was the fluorescence at the highest ligand concentration. $\mathrm{EC}_{50}$ was estimated using the Hill equation (Eq. 2). 
The Bahadur expansion was used to analyze the data ${ }^{36}$. Fitness for the bahadur expansion was defined as

$$
\text { fitness }_{\text {variant }}=\log _{10}\left(\frac{\text { fold induction }_{\text {variant }}}{\text { fold induction }_{\text {wildtype }}}\right)
$$

Fold induction in Eq. (2)was changed to basal gene expression, maximum gene expression, or $\mathrm{EC}_{50}$ for each functional parameter. Each mutant can be represented as a numerical string (z string), where each mutable position is one number $\left(z_{i}\right)$ in the string. A wild-type residue at a position is designated by a -1 while the mutated residue is designated by a 1 . The mutant M167L + F168Y thus becomes $[-1,-1,1,1]$. The interaction terms can be modeled as follows:

$$
\begin{gathered}
\varphi_{0}=1 \\
\varphi_{1}, \varphi_{2}, \ldots, \varphi_{n}=z_{1}, z_{2}, \ldots, z_{n} \\
\varphi_{n+1}, \varphi_{n+2}, \ldots, \varphi_{n+C_{2}^{n}}=z_{1} z_{2}, z_{1} z_{3}, \ldots, z_{n-1} z_{n} \\
\varphi_{2^{n}-1}=z_{1} z_{2} \ldots z_{n}
\end{gathered}
$$

An orthonormal matrix of psi-values is created based on the combinations of mutations within the set (Supplementary Table 4). The Bahadur coefficients can be calculated using this orthonormal matrix and a fluorescence values $f(x)$ for a particular mutant $x$ in the set of all mutants X.

$$
w_{i}=\frac{1}{2^{n}} \sum_{x \in X} f(x) \varphi_{i}(x)
$$

The fluorescence of each combinatorial mutant can be calculated based on the Bahadur coefficients and $\mathrm{z}$ string.

$$
f(x)=\sum_{i=0}^{2 n-1} w_{i} \varphi_{i}(x)
$$

The $R^{2}$ between the modeled fluorescence values and the experimental data is 1.0 when all interaction terms are included in the expansion. By truncating Eq. (9) to contain only low-order interactions, the effect of these contributions to the model can be determined. The expansion was applied to the full set of mutations (4 positions) and modeled using first-order terms; first- and second-order terms; first-, second-, and third-order terms; and all terms (Supplementary Fig. 19). An identical approach was applied to all 24 subnetworks and utilized only first-order terms in the reconstruction (Supplementary Fig. 20).

Errors in the $R^{2}$ statistics were estimated using a Monte Carlo simulation. 500 sets of fitness values for all mutants were sampled based on experimental fitness means and standard deviations following a Gaussian distribution using the NumPy module in Python $2.7^{45,46}$. Equations (8) and (9) were applied to reconstruct the fitness values and calculate $R^{2}$ values between the sampled model and the sampled data to give a distribution of $R^{2}$ values. Bias-corrected adjusted $95 \%$ confidence intervals were calculated by obtaining the average $R^{2}$ of 10,000 bootstrap iterations of the Monte Carlo simulation $R^{2}$. The bahadur expansion was applied to each functional parameter.

A control set of additive data was used to calculate the $R^{2}$ of data showing no epistasis (Supplementary Table 2). This control set was analyzed using the same approach as the subnetwork workflow.

Protein characterization. The $\operatorname{Ttg} R$ gene for variants $0000,1000,0100,1100$, and 1111 were cloned into a pET31B vector downstream of the T7 promoter for lacinducible transcription control using isothermal assembly with $0.18 \mathrm{pmol}$ backbone and $0.392 \mathrm{pmol} \operatorname{TtgR}$. MBP was amplified with primers to add a C-terminal His-tag and TEV site and inserted into the TtgR-pET31B vector upstream of TtgR to create a MBP-His-TtgR fusion with a TEV cleavage site between the His-tag and the TtgR protein. BL21 chemically competent cells (NEB) were transformed with $20 \mathrm{ng}$ of pET31B vector. Dilutions of transformants were plated on LB-ampicillin agar. A colony was selected and grown in $5 \mathrm{~mL}$ LB-ampicillin media shaking at $37^{\circ} \mathrm{C}$ for $16 \mathrm{~h}$. This culture was added to $500 \mathrm{~mL}$ autoinduction media (Terrific Broth, $0.8 \%$ glycerol, $2 \mathrm{mM} \mathrm{MgSO} 4,0.375 \%$ (w/v) aspartic acid, $0.015 \%(\mathrm{w} / \mathrm{v})$ glucose, $0.5 \%(\mathrm{w} / \mathrm{v})$ lactose) and grown for $8 \mathrm{~h}$ at $37^{\circ} \mathrm{C}$ shaking. The culture was grown for an additional $16 \mathrm{~h}$ at $25^{\circ} \mathrm{C}$ shaking.

The cells were spun down at $5500 \mathrm{~g}$ for $15 \mathrm{~min}$ at $4{ }^{\circ} \mathrm{C}$. The supernatant was removed and the cells were resuspended in a lysis buffer $(300 \mathrm{mM} \mathrm{NaCl}, 50 \mathrm{mM}$ HEPES, $1 \mathrm{mM}$ PMSF, $1 \mathrm{mg} / \mathrm{mL}$ Lysozyme, $5 \mathrm{mM}$ BME, 10\% glycerol, pH 7.5). A Q500 sonicator (Qsonica) was used to lyse cells using a $5 \mathrm{~s}$ on, $15 \mathrm{~s}$ off sonication protocol for $4 \mathrm{~min}$ total sonication time. The lysate was centrifuged at $14,000 \mathrm{~g}$ for $45 \mathrm{~min}$ at $4{ }^{\circ} \mathrm{C}$. The supernatant was isolated and filtered through a $0.22 \mu \mathrm{m}$ filter. The filtered supernatant was purified on an Akta Start using $25 \mathrm{~mL}$ HisTrap HP columns. The column was washed with 5 column volumes (CV) IMAC-A $(500 \mathrm{mM}$ $\mathrm{NaCl}, 20 \mathrm{mM}$ Imidazole, $20 \mathrm{mM}$ MOPS, $0.3 \mathrm{mM}$ TCEP, $\mathrm{pH}$ 7). MBP-6His-TtgR was eluted with a gradient of $100 \%$ IMAC-A to $100 \%$ IMAC-B $(500 \mathrm{mM} \mathrm{NaCl}$, $500 \mathrm{mM}$ Imidazole, $20 \mathrm{mM}$ MOPS, $0.3 \mathrm{mM}$ TCEP, pH7) over $5 \mathrm{CV}$ and collected in $2 \mathrm{~mL}$ fractions. Fractions with the highest absorbance at $280 \mathrm{~nm}$ (A280) were combined and dialyzed in $8 \mathrm{~L}$ of dialysis buffer A $(100 \mathrm{mM} \mathrm{NaCl}, 20 \mathrm{mM}$ MOPS, $0.3 \mathrm{mM}$ TCEP, $\mathrm{pH}$ 7.5). TEV was added to the proteins prior to dialysis at a ratio of
1:50 w/w TEV:TtgR. Dialysis occurred over a $16 \mathrm{~h}$ interval at $4{ }^{\circ} \mathrm{C}$ while stirring at low speed.

Dialyzed protein was centrifuged at $14,000 \mathrm{~g}$ for $10 \mathrm{~min}$ at $4{ }^{\circ} \mathrm{C}$. The supernatant was passed through a $0.22 \mu \mathrm{m}$ filter and loaded onto the HisTrap columns at $5 \mathrm{~mL} / \mathrm{min}$. The column was washed with $5 \mathrm{CV}$ of IMAC-A and $2 \mathrm{~mL}$ fractions were collected. $5 \mathrm{CV}$ of IMAC-B was used to remove the MBP-6His from the column. The column was washed with an additional 10CV IMAC-A. Wash fractions with high A280 were combined and reapplied to the column. The column was washed with $5 \mathrm{CV}$ of IMAC-A and $2 \mathrm{~mL}$ fractions were collected. 5CV of IMAC-B was used to strip the MBP-6His from the column. Fractions with high A280 were combined and dialyzed in $4 \mathrm{~L}$ of dialysis buffer $\mathrm{C}(100 \mathrm{mM} \mathrm{NaCl}, 20 \mathrm{mM}$ MOPS, $10 \mathrm{mM} \mathrm{MgCl} 2,0.3 \mathrm{mM}$ TCEP, $\mathrm{pH}$ 7.8). The protein was centrifuged at $14,000 \mathrm{~g}$ for $10 \mathrm{~min}$ at $4{ }^{\circ} \mathrm{C}$. The supernatant was passed through a $0.22 \mu \mathrm{m}$ filter. The protein was concentrated to approximately $9 \mathrm{mg} / \mathrm{mL}$ and frozen in $60 \mu \mathrm{L}$ aliquots in liquid nitrogen before storing at $-80^{\circ} \mathrm{C}$. Dialysis buffer $\mathrm{C}$ was passed through a $0.22 \mu \mathrm{m}$ filter and stored at $4{ }^{\circ} \mathrm{C}$ for ITC experiments.

Stocks of $250 \mathrm{mM}$ naringenin and $100 \mathrm{mM}$ resveratrol were diluted to $500 \mu \mathrm{M}$ and $250 \mu \mathrm{M}$, respectively, in dialysis buffer C. Aliquots of TtgR were thawed on ice and diluted to a final concentration of $7.5 \mu \mathrm{M}$. DMSO or ethanol was added to the TtgR solution to match the solution composition of the naringenin or resveratrol dilutions. An aliquot of dialysis buffer $\mathrm{C}$ was also prepared with DMSO or ethanol for a control injection and to wash the sample cell between ITC injections.

The ITC experiments were conducted on a VP-ITC (MicroCal). An initial control injection scheme consisted of loading the sample cell with dialysis buffer $\mathrm{C}$ and performing a series of $1010 \mu \mathrm{L}$ ligand injections with $10 \mathrm{~min}$ intervals at $25^{\circ} \mathrm{C}$. The sample cell was washed 5 times with dialysis buffer C before the $7.5 \mu \mathrm{M}$ protein solution was loaded. Twenty-five $10 \mu \mathrm{L}$ naringenin injections or $2810 \mu \mathrm{L}$ resveratrol injections occurred in $10 \mathrm{~min}$ intervals at $25^{\circ} \mathrm{C}$.

Data analysis was primarily conducted using Origin 7.0 (MicroCal). The heats of injection from the control sample were averaged. The protein-ligand injection profile was subtracted by this average heat prior to curve fitting. Due to low affinity for both naringenin and resveratrol, the stoichiometry of binding was fixed to 1 to reduce the degrees of freedom prior to fitting. The curves were fit with the single binding site model (Supplementary Fig. 21).

X-ray crystallography. TtgR-pET31B vector was electroporated into BL21 cells (NEB) and recovered in $1 \mathrm{~mL}$ SOC. The cells were incubated for $1 \mathrm{~h}$ at $37^{\circ} \mathrm{C}$ before serial dilutions were plated on LB-ampicillin $(100 \mu \mathrm{g} / \mathrm{mL})$ plates. A single colony was selected and incubated in $5 \mathrm{~mL}$ LB-ampicillin $(100 \mu \mathrm{g} / \mathrm{mL})$ at $37^{\circ} \mathrm{C}$ shaking for $3 \mathrm{~h}$. The $5 \mathrm{~mL}$ culture was added to $500 \mathrm{~mL}$ LB-ampicillin media and incubated at $37^{\circ} \mathrm{C}$ shaking at $250 \mathrm{rpm}$ for approximately $3 \mathrm{~h}$ until the $\mathrm{OD}_{600}$ reached 0.6 . The culture was induced with $100 \mu \mathrm{M}$ IPTG followed by an incubation at $16^{\circ} \mathrm{C}$ for $16 \mathrm{~h}$ shaking at $250 \mathrm{rpm}$.

The cells were spun down at $5500 \mathrm{~g}$ for $15 \mathrm{~min}$ at $4 \mathrm{C}$. The supernatant was removed and the cells were resuspended in a lysis buffer $(300 \mathrm{mM} \mathrm{NaCl}, 50 \mathrm{mM}$ HEPES, $1 \mathrm{mM}$ PMSF, $1 \mathrm{mg} / \mathrm{mL}$ Lysozyme, $5 \mathrm{mM}$ BME, $10 \%$ glycerol, $\mathrm{pH}$ 7.5). A Q500 sonicator (Qsonica) was used to lyse cells using a $25 \mathrm{~s}$ on, $50 \mathrm{~s}$ off sonication protocol for $3 \mathrm{~min}$ and $45 \mathrm{~s}$ total sonication time. The lysate was centrifuged at $14,000 \mathrm{~g}$ for $45 \mathrm{~min}$ at $4{ }^{\circ} \mathrm{C}$. The supernatant was isolated and filtered through a $0.22 \mu \mathrm{m}$ filter. The filtered supernatant was purified on an Akta Start (Cytiva) using $5 \mathrm{~mL}$ HisTrap HP columns (Cytiva). The supernatant was loaded onto the column at a flow rate of $5 \mathrm{~mL} / \mathrm{min}$. The column was washed with 5 column volumes (CV) IMAC-A. MBP-6His-TtgR was eluted with a gradient of $100 \%$ IMAC-A to $100 \%$ IMAC-B over $10 \mathrm{CV}$ and collected in $2 \mathrm{~mL}$ fractions. Fractions with the highest absorbance at $280 \mathrm{~nm}$ (A280) were combined and dialyzed in $8 \mathrm{~L}$ of dialysis buffer A. TEV was added to the proteins prior to dialysis at a ratio of 1:50 w/w TEV:TtgR. Dialysis occurred over a $16 \mathrm{~h}$ interval at $4{ }^{\circ} \mathrm{C}$ while stirring at low speed.

TtgR was isolated from MBP-6His through a subtractive IMAC protocol using the Akta Start and $5 \mathrm{~mL}$ HisTrap HP column. The dialyzed protein was centrifuged at $4000 \mathrm{~g}$ for $10 \mathrm{~min}$ at $4 \mathrm{C}$. Supernatant was passed through a $0.22 \mu \mathrm{m}$ filter and applied to the HisTrap column at $5 \mathrm{~mL} / \mathrm{min}$. 5CV IMAC-A was used to wash the column while $2 \mathrm{~mL}$ fractions were collected. 2.5CV IMAC-B was used to remove the MBP from the column and $5 \mathrm{~mL}$ fractions were collected. Wash fractions with high A280 were combined and dialyzed in $4 \mathrm{~L}$ of dialysis buffer B $(50 \mathrm{mM} \mathrm{NaCl}, 5 \mathrm{mM}$ MOPS, $0.3 \mathrm{mM}$ TCEP, $\mathrm{pH}$ 7.5). EDTA was added to the protein wash fractions to a final concentration of $10 \mathrm{mM}$ prior to dialysis. Dialysis occurred over a $16 \mathrm{~h}$ interval at $4 \mathrm{C}$ while stirring at low speed. TtgR was concentrated to $10 \mathrm{mg} / \mathrm{mL}$ using spin concentrators. Samples were spun at intervals of $3500 \mathrm{~g}$ for $5 \mathrm{~min}$ and mixed via pipette between spins. Concentrated $\mathrm{TtgR}$ was separated into $60 \mu \mathrm{L}$ aliquots and frozen in liquid nitrogen prior to storage at $-80^{\circ} \mathrm{C}$.

Samples of TtgR wild type and mutant proteins were received frozen in $5 \mathrm{mM}$ MOPS, pH 7.4, $50 \mathrm{mM} \mathrm{NaCl}, 0.3 \mathrm{mM}$ TCEP. Samples were thawed and centrifuged for $5 \mathrm{~min}$ at $21,130 \mathrm{~g}$. Sample supernatants were filtered with a 0.22 micron MillexGV syringe filter unit (Millipore) before applying to an equilibrated $10 \mathrm{~mm} \times 300 \mathrm{~mm}$ Superdex 200 column (GE Healthcare). Chromatography was performed on a GE AKTA FPLC system. Column buffer was $20 \mathrm{mM}$ HEPES, $\mathrm{pH}$ $7.5,350 \mathrm{mM} \mathrm{NaCl}, 0.3 \mathrm{mM}$ TCEP. Two primary peaks were obtained from each sample with major peak at approximately $45 \mathrm{kD}$ MW and a minor peak at approximately $79 \mathrm{kD}$. The fractions corresponding to the major peak were pooled and concentrated with an Amicon Ultracel-10 centrifugal filter device (Millipore) and dialyzed vs. $5 \mathrm{mM}$ HEPES, pH 7.5, $50 \mathrm{mM} \mathrm{NaCl}, 0.3 \mathrm{mM}$ TCEP. Samples 
collected after dialysis were divided into small aliquots and flash frozen in PCR tubes with liquid nitrogen.

Crystallization screening and optimization were conducted in the Collaborative Crystallography Core in the Department of Biochemistry and the University of Wisconsin-Madison. Crystallization experiments were set up using a SPT Labtech mosquito ${ }^{\circledast}$ crystallization robot in MRC SD-2 crystallization plates at $4{ }^{\circ} \mathrm{C}$ and $20^{\circ} \mathrm{C}$ $(277$ and $293 \mathrm{~K}$.) Crystals progressing to diffraction experiments were all obtained at $20^{\circ} \mathrm{C}$. Two general screens, Hampton Research IndexHT and Molecular Dimensions JCSG+ were used in this study ${ }^{47}$. Crystals were detected using brightfield and UV fluorescence imaging with a JANSi UVEX-P crystallization plate imaging system supplementing visual inspection with stereomicroscopes. Initial rounds of crystallization optimization were performed in SD2 plates using the mosquito to expand 24 solution conditions by setting columns of experiments in four different samples to reservoir volume ratios. Cryoprotected crystals were harvested in Mitegen micro mounts and flash cooled by immersion in liquid nitrogen.

Crystals were screened and X-ray diffraction data were collected at Advanced Photon Source (APS) beamlines LS-CAT and GM/CA@APS, universally on crystals cooled to $100 \mathrm{~K}$. Diffraction data was reduced using XDS (VERSION Mar 15, 2019 BUILT $=20190315)$ and scaled with XSCALE (VERSION Mar 15, 2019 BUILT $=20190315)^{48,49}$. Structures were solved by molecular replacement with Phaser V2.8.2 within the Phenix suite of programs (V1.18.2_3874), automatically rebuilt with phenix.autobuild, iteratively improved with alternating rounds of rebuilding in Coot and refinement using phenix.refine, and validated using MOLPROBITY V4.02-528 $50-54$

7K1A. Crystals providing diffraction data were grown by mixing $200 \mathrm{~nL}$ of protein at $9.7 \mathrm{mg} / \mathrm{mL}$ in sample buffer (5 mM HEPES pH 7.5, $50 \mathrm{mM} \mathrm{NaCl}, 0.3 \mathrm{mM}$ TCEP) with $150 \mathrm{~nL}$ of reservoir solution, was equilibrated against $150 \mathrm{~nL} 20 \%$ MEPEG, $0.2 \mathrm{M} \mathrm{MgCl}_{2}, 0.1 \mathrm{M}$ bistris $\mathrm{HCl} \mathrm{pH} 6.5$ equilibrated against $50 \mu \mathrm{L}$ of reservoir solution in a SD2 plate. Samples were cryoprotected with reservoir solution supplemented to $35 \%$ MEPEG 2000. A $360^{\circ}$ sweep of data (720 frames) was collected on a MAR 300 CCD detector at LS-CAT beamline 21ID-G on 2018-12-16 using $0.97856 \AA$ X-rays. The phase problem was solved using $2 \mathrm{UXU}(\mathrm{A})$ as a molecular replacement model $^{28}$.

7K1C. Crystals of wild-type TtgR with resveratrol were prepared by incubating $0.41 \mathrm{mM}$ protein $(9.8 \mathrm{mg} / \mathrm{mL})$ and $0.5 \mathrm{mM}$ resveratrol dissolved in sample buffer for $30 \mathrm{~min}$ at room temperature prior to setting up crystallization experiments. The crystal yielding the best diffraction data were grown by mixing $200 \mathrm{~nL}$ of the protein-ligand sample with $250 \mathrm{~nL}$ reservoir (18\% PEG4000, 0.2 $\mathrm{M} \mathrm{MgCl}_{2}, 0.1 \mathrm{M}$ bistris $\mathrm{HCl} \mathrm{pH} \mathrm{6.5)} \mathrm{equilibrated} \mathrm{against} 50 \mu \mathrm{L}$ of reservoir a SD2 plate. Samples were cryoprotected with reservoir solution supplemented with 35\% PEG4000. A $360^{\circ}$ sweep of data (720 frames) was collected on a MAR 300 CCD detector at LSCAT beamline 21ID-G on 2018-12-16 using $0.97856 \AA$ X-rays. The phase problem was solved using $2 \mathrm{XDN}$ as a molecular replacement model.

7KD8. Crystals were prepared by incubating $0.43 \mathrm{mM}(10.4 \mathrm{mg} / \mathrm{mL})$ quadruple mutant protein with $1 \mathrm{mM}$ resveratrol in sample buffer for $30 \mathrm{~min}$ prior to setting up crystallization experiments. Crystals providing the reported diffraction data set grew from $2 \mu \mathrm{L}$ of sample mixed with $2 \mu \mathrm{L}$ of reservoir solution (12\% MEPEG 2000, 5\% 2-methyl-2,4-pentanediol, $0.3 \mathrm{M} \mathrm{MgCl}_{2}, 0.1 \mathrm{M}$ bistris buffer at $\mathrm{pH} 6.5$ equilibrated in a hanging drop experiment using a siliconized glass cover slip. Samples were cryoprotected with reservoir solution supplemented to $30 \%$ MEPEG 2000 . A $360^{\circ}$ (3600 frames) shutterless data set was collected at LS-CAT 21ID-D on 2019-05-30 with an Eiger $9 \mathrm{M}$ direct detector and $1.07812 \AA \mathrm{X}$-rays. The phase problem was solved using $7 \mathrm{~K} 1 \mathrm{~A}$ as a molecular replacement model.

Reporting summary. Further information on research design is available in the Nature Research Reporting Summary linked to this article.

\section{Data availability}

The datasets generated and analyzed during the current study are available from the corresponding author on reasonable request. The crystallography data generated in this study are available in the RCSB under the following accession codes: $7 \mathrm{~K} 1 \mathrm{~A}, 7 \mathrm{~K} 1 \mathrm{C}$, and 7KD8. The naringenin-bound wild-type TtgR structure data used in this study are available in the RCSB Protein Data Bank under accession code 2UXU. The minocyclinebound TtgR structure data used for computational design are available in the RCSB Protein Data Bank under accession code $2 \mathrm{UXH}$. Source data are provided with this paper.

\section{Code availability}

All figures were generated using the Matplotlib module in Python 2.755. Python and shell scripts for generating input from Rosetta and analyzing from Rosetta are available at: https://github.com/raman-lab/biosensor_design. Scripts used in data analysis of flow cytometry data, epistasis analysis, and figure generation can be found at: https:// github.com/raman-lab/epistasis. Flow cytometry data were analyzed in FlowJo V10. ITC data were analyzed using Origin V7.0. POVME V3.0 was used to calculate pocket volumes based on the location of resveratrol ${ }^{56}$.

Received: 29 November 2020; Accepted: 3 September 2021; Published online: 21 September 2021

\section{References}

1. Breen, M. S., Kemena, C., Vlasov, P. K., Notredame, C. \& Kondrashov, F. A. Epistasis as the primary factor in molecular evolution. Nature 490, 535-538 (2012).

2. Smith, J. M. Natural selection and the concept of a protein space. Nature $\mathbf{2 2 5}$ 2 (1970).

3. Lunzer, M., Golding, G. B. \& Dean, A. M. Pervasive cryptic epistasis in molecular evolution. PLOS Genet. 6, e1001162 (2010).

4. Gong, L. I., Suchard, M. A. \& Bloom, J. D. Stability-mediated epistasis constrains the evolution of an influenza protein. Elife 2, e00631 (2013).

5. Wu, N. C., Dai, L., Olson, C. A., Lloyd-Smith, J. O. \& Sun, R. Adaptation in protein fitness landscapes is facilitated by indirect paths. Elife 5, https:// doi.org/10.7554/eLife.16965 (2016).

6. Miton, C. M., Buda, K. \& Tokuriki, N. Epistasis and intramolecular networks in protein evolution. Curr. Opin. Struct. Biol. 69, 160-168 (2021).

7. Starr, T. N. \& Thornton, J. W. Epistasis in protein evolution. Protein Sci. 25, 1204-1218 (2016).

8. McKeown, A. N. et al. Evolution of DNA specificity in a transcription factor family produced a new gene regulatory module. Cell 159, 58-68 (2014).

9. Anderson, D. W., McKeown, A. N. \& Thornton, J. W. Intermolecular epistasis shaped the function and evolution of an ancient transcription factor and its DNA binding sites. Elife 4, e07864 (2015).

10. Dickinson, B. C., Leconte, A. M., Allen, B., Esvelt, K. M. \& Liu, D. R. Experimental interrogation of the path dependence and stochasticity of protein evolution using phage-assisted continuous evolution. Proc. Natl Acad. Sci. USA 110, 9007-9012 (2013).

11. Podgornaia, A. I. \& Laub, M. T. Pervasive degeneracy and epistasis in a protein-protein interface. Science 347, 5 (2015).

12. Kaltenbach, M., Jackson, C. J., Campbell, E. C., Hollfelder, F. \& Tokuriki, N. Reverse evolution leads to genotypic incompatibility despite functional and active site convergence. Elife 4, https://doi.org/10.7554/eLife.06492 (2015).

13. Lunzer, M., Miller, S. P., Felsheim, R. \& Dean, A. M. The biochemical architecture of an ancient adaptive landscape. Science 310, 3 (2005).

14. Wilson, C. et al. Using ancient protein kinases to unravel a modern cancer drug's mechanism. Science 347, 5 (2015).

15. Starr, T. N., Picton, L. K. \& Thornton, J. W. Alternative evolutionary histories in the sequence space of an ancient protein. Nature 549, 409-413 (2017).

16. Olson, C. A., Wu, N. C. \& Sun, R. A comprehensive biophysical description of pairwise epistasis throughout an entire protein domain. Curr. Biol. 24, 2643-2651 (2014)

17. Hart, K. M. et al. Thermodynamic system drift in protein evolution. PLoS Biol. 12, 8 (2014).

18. Risso, V. A., Gavira, J. A., Mejia-Carmona, D. F., Gaucher, E. A. \& Sanchez-Ruiz, J. M. Hyperstability and substrate promiscuity in laboratory resurrections of Precambrian beta-lactamases. J. Am. Chem. Soc. 135, 2899-2902 (2013).

19. Patel, M. P. et al. Synergistic effects of functionally distinct substitutions in beta-lactamase variants shed light on the evolution of bacterial drug resistance. J. Biol. Chem. 293, 17971-17984 (2018).

20. Bershtein, S., Segal, M., Bekerman, R., Tokuriki, N. \& Tawfik, D. S. Robustness-epistasis link shapes the fitness landscape of a randomly drifting protein. Nature 444, 929-932 (2006).

21. Tomatis, P. E. et al. Adaptive protein evolution grants organismal fitness by improving catalysis and flexibility. Proc. Natl Acad. Sci. USA 105, 6 (2008).

22. Bloom, J. D., Labthavikul, S. T., Otey, C. R. \& Arnold, F. H. Protein stability promotes evolvability. Proc. Natl Acad. Sci. USA 103, 5 (2006).

23. Wodak, S. J. et al. Allostery in its many disguises: from theory to applications. Structure 27, 566-578 (2019).

24. Motlagh, H. N., Wrabl, J. O., Li, J. \& Hilser, V. J. The ensemble nature of allostery. Nature 508, 331-339 (2014).

25. Eick, G. N., Colucci, J. K., Harms, M. J., Ortlund, E. A. \& Thornton, J. W Evolution of minimal specificity and promiscuity in steroid hormone receptors. PLoS Genet. 8, e1003072 (2012).

26. Lisi, G. P., East, K. W., Batista, V. S. \& Loria, J. P. Altering the allosteric pathway in IGPS suppresses millisecond motions and catalytic activity. Proc. Natl Acad. Sci. USA 114, E3414-E3423 (2017).

27. Skerker, J. M. et al. Rewiring the specificity of two-component signal transduction systems. Cell 133, 1043-1054 (2008).

28. Alguel, Y. et al. Crystal structures of multidrug binding protein TtgR in complex with antibiotics and plant antimicrobials. J. Mol. Biol. 369, 829-840 (2007). 
29. Teran, W. et al. Antibiotic-dependent induction of Pseudomonas putida DOT-T1E TtgABC efflux pump is mediated by the drug binding repressor TtgR. Antimicrobial. Agents Chemother. 47, 3067-3072 (2003).

30. Daniels, C., Daddaoua, A., Lu, D., Zhang, X. \& Ramos, J. L. Domain cross-talk during effector binding to the multidrug binding TTGR regulator. J. Biol. Chem. 285, 21372-21381 (2010).

31. Teran, W., Krell, T., Ramos, J. L. \& Gallegos, M. T. Effector-repressor interactions, binding of a single effector molecule to the operator-bound $\mathrm{TtgR}$ homodimer mediates derepression. J. Biol. Chem. 281, 7102-7109 (2006).

32. Fleishman, S. J. et al. RosettaScripts: a scripting language interface to the Rosetta macromolecular modeling suite. PLoS ONE 6, e20161 (2011).

33. Xiong, D. et al. Improving key enzyme activity in phenylpropanoid pathway with a designed biosensor. Metab. Eng. 40, 115-123 (2017).

34. Leaver-Fay, A. et al. Scientific benchmarks for guiding macromolecular energy function improvement. Methods Enzymol. 523, 109-143 (2013).

35. Taylor, N. D. et al. Engineering an allosteric transcription factor to respond to new ligands. Nat. Methods 13, 177-183 (2016).

36. Matsuura, T., Kazuta, Y., Aita, T., Adachi, J. \& Yomo, T. Quantifying epistatic interactions among the components constituting the protein translation system. Mol. Syst. Biol. 5, 297 (2009)

37. Leaver-Fay, A. et al. ROSETTA3: an object-oriented software suite for the simulation and design of macromolecules. Methods Enzymol. 487, 545-574 (2011).

38. Nivon, L. G., Moretti, R. \& Baker, D. A pareto-optimal refinement method for protein design scaffolds. PLoS ONE 8, e59004 (2013)

39. Siegel, J. B. et al. Computational design of an enzyme catalyst for a stereoselective bimolecular Diels-Alder reaction. Science 329, 309-313 (2010).

40. Olins, P. O., Devine, C. S., Rangwala, S. H. \& Kavka, K. S. The T7 phage gene 10 leader RNA, a ribosome-binding site that dramatically enhances the expression of foreign genes in Escherichia coli Gene 73, 9 (1988).

41. Gibson, D. G. et al. Enzymatic assembly of DNA molecules up to several hundred kilobases. Nat. Methods 6, 343-345 (2009).

42. Kosuri, S. et al. Composability of regulatory sequences controlling transcription and translation in Escherichia coli. Proc. Natl Acad. Sci. USA 110, 14024-14029 (2013).

43. Hill, A. V. The possible effects of the aggregation of the molecules of haemoglobin on its dissociation curves. J. Physiol. (Lond.) 40, iv-vii (1910).

44. Virtanen, P. et al. SciPy 1.0: fundamental algorithms for scientific computing in Python. Nat. Methods 17, 261-272 (2020).

45. Oliphant, T. E. A Guide to NumPy. (Trelgol Publishing, 2006).

46. Walt, S. V. D., Colbert, S. C. \& Varoquaux, G. The NumPy array: a structure for efficient numerical computation. Comput. Sci. Eng. 13, 22-30 (2011).

47. Page, R. et al. Shotgun crystallization strategy for structural genomics: an optimized two-tiered crystallization screen against the Thermotoga maritima proteome. Acta Crystallogr. D 59, 1028-1037 (2003).

48. Kabsch, W. XDS. Acta Crystallogr. D 66, 125-132 (2010).

49. Diederichs, K., McSweeney, S. \& Ravelli, R. B. G. Zero-dose extrapolation as part of macromolecular synchrotron data reduction. Acta Crystallogr. D 59, 903-909 (2003).

50. Liebschner, D. et al. Macromolecular structure determination using X-rays, neutrons and electrons: recent developments in Phenix. Acta Crystallogr. D Struct. Biol. 75, 861-877 (2019).

51. Terwilliger, T. S. O. L. V. E. and RESOLVE: automated structure solution, density modification and model building. J. Synchrotron Radiat. 11, 49-52 (2003).

52. Emsley, P., Lohkamp, B., Scott, W. G. \& Cowtan, K. Features and development of Coot. Acta Crystallogr. D: Biol. Crystallogr. 66, 486-501 (2010).

53. Afonine, P. V. et al. Towards automated crystallographic structure refinement with phenix. refine. Acta Crystallogr. D: Biol. Crystallogr. 68, 352-367 (2012).

54. Chen, V. B. et al. MolProbity: all-atom structure validation for macromolecular crystallography. Acta Crystallogr. D: Biol. Crystallogr. 66, 12-21 (2010).

55. Hunter, J. D. Matplotlib: A 2D graphics environment. Comput. Sci. Eng. 9, 90-95 (2007).
56. Wagner, J. R. et al. POVME 3.0: software for mapping binding pocket flexibility. J. Chem. Theory Comput. 13, 4584-4592 (2017).

\section{Acknowledgements}

The authors thank Dr. Tina Wang, Dr. Phil Romero and Phil Huss for critical review of the manuscript. This work was supported by is partially supported by the US Army Research Office (W911NF-17-1-0043). This research was performed using the compute resources and assistance of the UW-Madison Center for High Throughput Computing (CHTC) in the Department of Computer Sciences. The CHTC is supported by UWMadison, the Advanced Computing Initiative, the Wisconsin Alumni Research Foundation, the Wisconsin Institutes for Discovery, and the National Science Foundation, and is an active member of the Open Science Grid, which is supported by the National Science Foundation and the U.S. Department of Energy's Office of Science. This research used resources of the Advanced Photon Source, a U.S. Department of Energy (DOE) Office of Science User Facility operated for the DOE Office of Science by Argonne National Laboratory under Contract No. DE-AC02-06CH11357. Use of the LS-CAT Sector 21 was supported by the Michigan Economic Development Corporation and the Michigan Technology Tri-Corridor (Grant 085P1000817). We thank Spencer Anderson, Joseph Brunzelle, Elena Kondrashkina, and Zdzislaw Wawrzak (LS-CAT) and Craig Ogata, Michael Becker and Nagarajan Venugopalan (GM/CA@APS) for synchrotron beamline support. K.N is supported by NIH National Research Service Award T32 GM07215 and the Robert and Katherine Burris Biochemistry Fund.

\section{Author contributions}

K.N. and S.R. designed the study, analyzed the data, and wrote the manuscript. K.N. performed all experiments. N.H. carried out computational design. R.S. and C.B. purified and crystallized the proteins.

\section{Competing interests}

The authors declare no competing interests.

\section{Additional information}

Supplementary information The online version contains supplementary material available at https://doi.org/10.1038/s41467-021-25826-7.

Correspondence and requests for materials should be addressed to Srivatsan Raman.

Peer review information Nature Communications thanks the anonymous reviewer(s) for their contribution to the peer review of this work.

Reprints and permission information is available at http://www.nature.com/reprints

Publisher's note Springer Nature remains neutral with regard to jurisdictional claims in published maps and institutional affiliations.

pen Access This article is licensed under a Creative Commons Attribution 4.0 International License, which permits use, sharing, adaptation, distribution and reproduction in any medium or format, as long as you give appropriate credit to the original author(s) and the source, provide a link to the Creative Commons license, and indicate if changes were made. The images or other third party material in this article are included in the article's Creative Commons license, unless indicated otherwise in a credit line to the material. If material is not included in the article's Creative Commons license and your intended use is not permitted by statutory regulation or exceeds the permitted use, you will need to obtain permission directly from the copyright holder. To view a copy of this license, visit http://creativecommons.org/ licenses/by/4.0/.

(C) The Author(s) 2021 